\title{
Analysis of the Relative Dynamics of a Charged Spacecraft Moving under the Influence of a Magnetic Field
}

\author{
Yu Cheng ${ }^{\mathrm{a}, \mathrm{b}}$, Gerard Gómez ${ }^{\mathrm{b}}$, Josep J. Masdemont ${ }^{\mathrm{c}}$, Jianping Yuan ${ }^{\mathrm{a}, *}$ \\ ${ }^{a}$ National Key Laboratory of Aerospace Flight Dynamics, Northwestern Polytechnical \\ University, 127 West Youyi Road, Xi'an, 710072, China \\ ${ }^{b}$ IEEC \& Department de Matemàtiques i Informàtica, Universitat de Barcelona, Gran Via \\ 585, 08007 Barcelona, Spain \\ ${ }^{c}$ IEEC \& Department de Matemàtiques, Universitat Politècnica de Catalunya, Diagonal \\ 647, 08028 Barcelona, Spain
}

\begin{abstract}
We analyse a dynamical scenario where a constantly charged spacecraft (follower) moves in the vicinity of another one (leader) that follows a circular Keplerian orbit around the Earth and generates a rotating magnetic dipole. The mass of the follower is assumed to be negligible when compared with the one of the leader and they are supposed to be in a high-Earth orbit, so the Lorentz force on the follower due to the geomagnetic field is ignored. With these assumptions, the motion of the leader is not perturbed by the follower and it is only subjected to the Earth's gravitational force field, while the charged follower is under to both the gravitational force of the Earth and the Lorentz force due to the magnetic dipole of the leader.

We focus on the dynamical characteristics of the system as a function of its parameters, with special attention to the ratio of the leader's mean motion around the Earth to the rotating rate of the dipole. We study the critical points of the model and their stability, the admissible and forbidden regions of motion of the deputy using the zero velocity surfaces and the families of periodic orbits emanating from equilibria. In the normal case we pay special attention to the
\end{abstract}

\footnotetext{
${ }^{*}$ Corresponding author

Email address: jyuan@nwpu.edu.cn (Jianping Yuan )
} 
periodic orbits of elliptic type and to the families of $2 \mathrm{D}$ tori surrounding them that are computed by means of a parameterisation method. The result is a fine catalog of orbits together with an accurate dynamical description suitable to researchers interested in potential applications of satellite formation flight using this kind of technology.

Keywords: Relative Dynamics, Periodic Orbits, Invariant Tori, Lorentz Force, Stability Analysis

\section{Introduction}

When a charged spacecraft moves in a magnetic field it experiences a Lorentz force. This propellant-less force, which is perpendicular to both the instantaneous velocity of the spacecraft and the magnetic field, offers some advantages

5 when compared with the traditional chemical propulsion and is a promising technology for future space missions. The magnetic dipole of the leader is supposed to be produced by three concentric and orthogonal high-temperature superconducting coils (HTSC) [29, in such a way that it can point along any direction adjusting the current in the three HTSC. Here we will only consider three possible orientations: normal, radial and tangential, according to its relative position in the orbital plane of the leader.

Previous works in astrodynamics applications of Lorentz forces have mainly considered the effect of natural magnetic fields on a charged spacecraft, such as the geomagnetic field [17, 45, 27, or the magnetic filed of other planets [35] on the spacecraft. Some of the applications that have been studied include the determination of new synchronous orbits using the geomagnetic field [40, the use of the Lorentz force as a means for orbit control and formation flying [28, 43], the effect of this force in a Jovian orbit insertion [2] as well as in gravity-assist manoeuvres [42]. Several control strategies using Lorentz force have also been considered for different kinds of missions [38, 41, 20] .

Using artificial magnetic fields, Kong [22] introduced the idea of Electro Magnetic Formation Flight (EMFF), which uses the intersection between the 
electromagnetic fields of several satellites to control the configuration of the formation; Umair [44] designed a control strategy for electromagnetic satellite formations in near-Earth orbits; Kwon 23 explored the applicability of EMFF for attitude and translation control of close proximity formation flying, and Porter [32] demonstrated the feasibility of electromagnetic formation flight in a micro-gravity environment.

Inspired by previous works, a new dynamical scenario was proposed by Peng 30, where a charged follower moves around a leader spacecraft which produces a rotating magnetic dipole pointing along the radial direction defined by the Earth and the leader. In [31] Peng extended the previous work to study the planar periodic orbits, suitable for formation flying, when the magnetic dipole is perpendicular to the orbital plane of the leader. Our paper aims to extend 35 the work done by Peng considering three possible orientations of the dipole: normal, radial and tangential and pursuing a fine dynamical analysis in all the cases.

From the engineering point of view, there are some technical questions about the realisation of the system that must be considered. They are mainly related 40 to the thermal control system of the HTSC, and the satellite charging of the follower. They have been studied, among others, by Kwon [23], who designed a cryogenic heat pipe to cool the HTSC, Baudouy [7, who surveyed the main cooling techniques at low temperature, and Saaj et al., that showed that current technology can realise charge-to-mass ratio of the order of $10^{-6}$ to $10^{-3}$.

Although the device required to generate a steerable electromagnetic dipole, using, for instance, three orthogonal electromagnetic coils made of superconducting wires, is a massive structure, we will assume that the gravitational interaction between the leader and the follower is negligible, at least when it is compared with the one due to the Lorentz force.

50 The above mentioned technical questions are out of the scope of this paper. Our main attention is to focus on the dynamical analysis of the model, giving an accurate description suitable for the exploration of possible applications. The analysis and numerical simulations will be based on the assumption that all the 
proposed magnetic field or charge-to-mass ratio can be satisfied which, although might be beyond the current state-of-art technology, we hope that can be reality in the near future.

The paper is organised as follows: in Section 2 the dynamical model is introduced, its symmetry properties, and the differentail equations of the three different cases considered according to the orientation of the magnetic dipole: normal, radial and tangential. Each one of these cases is studied in Sections 3 . 4 and, 5 , respectively. For each case we have computed the equilibrium points and its evolution with one of the parameters of the model, the zero velocity surfaces that determine the allowable regions of motion in the configuration space, and the periodic orbits emanaiting from the different equilibria. In the normal case, we have also determined three families of $2 \mathrm{D}$ invariant tori with a fixed energy value. The methods used for the computation of the symmetric and nonsymmetric periodic orbits emanating from equilibria, and the parameterisation method for the numerical computation of the 2D invariant tori are given in the Appendix (Section 7). In the last section we give some conclusions. Possible applications of the obtained results to formation flying, mainly the ones related to the use of some of the periodic orbits determined as nominal trajectories, will be discussed in a forthcoming paper.

\section{Modelling equations and symmetries}

As it has been mentioned, we assume that the mass of the follower is negli75 gible when compared with the one of the leader, which is assumed to move in a circular high-Earth orbit (such as a GEO), so that the geomagnetic Lorentz force on the follower is ignored. As a consequence, the follower is subjected to both the gravitational force of the Earth and the Lorentz force due to the artificial magnetic dipole. As it is usual in the description of relative motions, we use a local-vertical-local-horizontal (LVLH) reference system, with the origin located on the leader, the positive $x$-axis $\left(\boldsymbol{e}_{r}\right)$ pointing from the Earth to the leader, the $z$-axis $\left(\boldsymbol{e}_{n}\right)$ parallel to the leader's angular momentum, and the 
$y$-axis $\left(\boldsymbol{e}_{t}\right)$ completing a right-hand coordinate system (see Fig. 1).

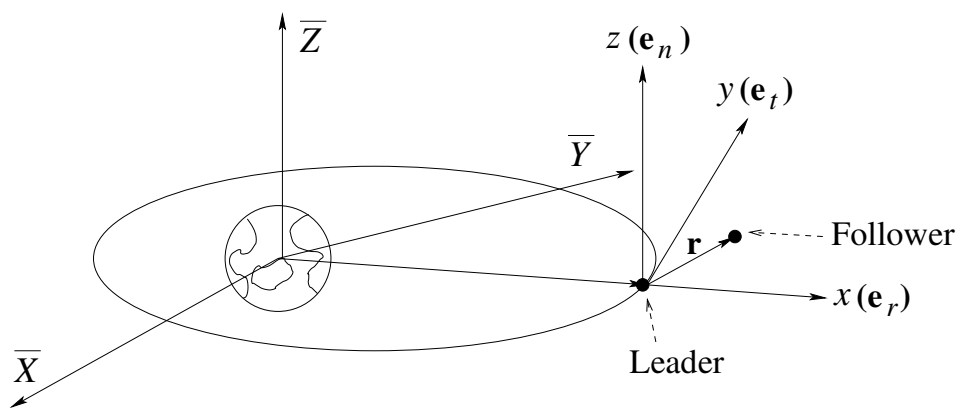

Figure 1: The local-vertical-local-horizontal reference system $\left(\boldsymbol{e}_{r}, \boldsymbol{e}_{n}, \boldsymbol{e}_{T}\right)$.

In this LVLH reference system, the equations of relative motion of the follower with respect to the leader can be written as a perturbation of the HillClohessy-Wiltshire (HCW) equations in the following way:

$$
\begin{aligned}
\ddot{x}-2 n \dot{y}-3 n^{2} x & =f_{x}, \\
\ddot{y}+2 n \dot{x} & =f_{y}, \\
\ddot{z}+n^{2} z & =f_{z},
\end{aligned}
$$

where $n$ is the mean motion of the circular orbit of the leader around the Earth, and $\left(f_{x}, f_{y}, f_{z}\right)^{T}$ are the three components of the Lorentz force $\boldsymbol{f}_{L}$ acting on the follower.

It must be noted that if the Lorentz force $f_{L}$ vanishes, then (1) become the usual HCW equations, which have been extensively studied in the literature (see, for instance, [8, 15], and [33]). In this case, the dynamics of the problem is 90 much simpler, since the HCW equations describe the relative dynamics of two Keplerian orbits, that is far from the problem considered in this paper, in which the Lorentz force has a key role.

The value of the Lorentz force is given by:

$$
\boldsymbol{f}_{L}=\frac{q}{m} \boldsymbol{v}_{r} \times \boldsymbol{B}=\frac{q}{m} \cdot\left(\dot{\boldsymbol{r}}-\boldsymbol{\omega}_{c} \times \boldsymbol{r}\right) \times \boldsymbol{B} .
$$

Here $q$ denotes the charge of the follower, $m$ its mass, $\boldsymbol{v}_{r}$ the relative velocity of the follower with respect to the rotating magnetic field, $\boldsymbol{B}$ the magnetic rotating 
field, $\boldsymbol{r}$ and $\dot{\boldsymbol{r}}$ the position and velocity of the follower in the LVLH reference frame, and $\boldsymbol{\omega}_{c}$ the dipole's rotational velocity. If $\omega_{c}$ is the modulus of $\boldsymbol{\omega}_{c}$, we can write

$$
\boldsymbol{\omega}_{c}=\omega_{c}\left(N_{x}, N_{y}, N_{z}\right)^{T},
$$

where $\boldsymbol{N}=\left(N_{x}, N_{y}, N_{z}\right)^{T}$ is the unitary vector in the direction of $\boldsymbol{B}$.

The magnetic field $\boldsymbol{B}$ is defined as the curl of the potential $\boldsymbol{A}(\boldsymbol{B}=\nabla \times \boldsymbol{A})$, with $\boldsymbol{A}$ given by :

$$
\boldsymbol{A}=\frac{B_{0}}{r^{2}}(\boldsymbol{N} \times \hat{\boldsymbol{r}})=\frac{B_{0}}{r^{3}}\left[\begin{array}{lll}
\left.z N_{y}-y N_{z}\right) & \left(x N_{z}-z N_{x}\right) & \left(y N_{x}-x N_{y}\right)
\end{array}\right]^{T}
$$

where $r=\|\boldsymbol{r}\|=\sqrt{x^{2}+y^{2}+z^{2}}$ is the leader -follower distance, $\hat{\boldsymbol{r}}=(\hat{x}, \hat{y}, \hat{z})^{T}=$ $\boldsymbol{r} / r$, and $B_{0}$ is the magnetic dipole moment, with units: $\mathrm{Wb} \cdot \mathrm{m}$ (see [23]), which for a coil is defined by

$$
B_{0}=\frac{\mu_{0}}{4 \pi} n_{c} i_{c} \pi r_{c}^{2},
$$

where $\mu_{0}=4 \pi \times 10^{-7} \mathrm{~N} / \mathrm{A}^{2}$ is the vacuum permeability, $n_{c}$ the number of loops in the coil, $i_{c}$ the current flown intensity, and $r_{c}$ the radius of the coil (see [23]). Once the size of the coil $\left(n_{c}\right.$ and $\left.r_{c}\right)$ is fixed, the magnetic dipole moment is determined by the value of current $i_{c}$ passing through, which, according to the material of the coil and working temperature, is limited by the current density of the coil. In our mathematical analysis we assume that the coil can carry enough current to produce the required magnetic moment.

Using the above notation, the components of the Lorentz force $\boldsymbol{f}_{L}$ can be written as,

$$
\begin{aligned}
& f_{x}=\frac{q}{m} \frac{B_{0}}{r^{3}}\left[3(\boldsymbol{N} \cdot \hat{\boldsymbol{r}})(\dot{y} \hat{z}-\dot{z} \hat{y})-\omega_{c}\left(x N_{z}-z N_{x}\right)\left(3(\boldsymbol{N} \cdot \hat{\boldsymbol{r}}) \hat{z}-N_{z}\right)\right. \\
& \left.+\dot{z} N_{y}-\dot{y} N_{z}+\omega_{c}\left(y N_{x}-x N_{y}\right)\left(3(\boldsymbol{N} \cdot \hat{\boldsymbol{r}}) \hat{y}-N_{y}\right)\right], \\
& f_{y}=\frac{q}{m} \frac{B_{0}}{r^{3}}\left[3(\boldsymbol{N} \cdot \hat{\boldsymbol{r}})(\dot{z} \hat{x}-\dot{x} \hat{z})-\omega_{c}\left(y N_{x}-x N_{y}\right)\left(3(\boldsymbol{N} \cdot \hat{\boldsymbol{r}}) \hat{x}-N_{x}\right)\right. \\
& \left.+\dot{x} N_{z}-\dot{z} N_{x}+\omega_{c}\left(z N_{y}-y N_{z}\right)\left(3(\boldsymbol{N} \cdot \hat{\boldsymbol{r}}) \hat{z}-N_{z}\right)\right], \\
& f_{z}=\frac{q}{m} \frac{B_{0}}{r^{3}}\left[3(\boldsymbol{N} \cdot \hat{\boldsymbol{r}})(\dot{x} \hat{y}-\dot{y} \hat{x})-\omega_{c}\left(z N_{y}-y N_{z}\right)\left(3(\boldsymbol{N} \cdot \hat{\boldsymbol{r}}) \hat{y}-N_{y}\right)\right. \\
& \left.+\dot{y} N_{x}-\dot{x} N_{y}+\omega_{c}\left(x N_{z}-z N_{x}\right)\left(3(\boldsymbol{N} \cdot \hat{\boldsymbol{r}}) \hat{x}-N_{x}\right)\right] .
\end{aligned}
$$


We note that, in principle, the three orthogonal super-conducting wires can produce the magnetic field in any direction, but in what follows we will only consider the following three dipole orientations:

1. Normal case. $\boldsymbol{B}$ is aligned with $\boldsymbol{N}=(0,0, \pm 1)^{T}$ : the dipole axis is parallel to $\boldsymbol{e}_{n}$.

2. Radial case. $\boldsymbol{B}$ is aligned with $\boldsymbol{N}=( \pm 1,0,0)^{T}$ : the dipole axis is parallel to the radius vector $\boldsymbol{e}_{r}$.

3. Tangential case. $\boldsymbol{B}$ is aligned with $\boldsymbol{N}=(0, \pm 1,0)^{T}$ : the dipole axis is parallel to $e_{t}$.

Following again [30, and in order to simplify the equations of motion, we introduce a set of non-dimensional units:

- Time unit: $\tau=n t$. The derivative with respect to $\tau$ will be denoted by a prime, and clearly

$$
\frac{d}{d \tau}=n \frac{d}{d t}
$$

- Length unit: $\alpha$ such that

$$
\alpha^{3}=\left|\frac{q}{m} B_{0} \frac{\omega_{c}}{n^{2}}\right|=\left|\frac{q}{m} B_{0} \frac{1}{n \beta}\right|
$$

where the parameter $\beta$ is defined by $\beta=n / \omega_{c}$.

Table 1 lists all the parameters that have already been introduced. In the three cases associated to the three dipole orientation, and using a nondimensional set of units, we will allow to vary only two: the motion angular velocity ration $\beta$, and the sign $\sigma$ of the charge $q$.

\subsection{Differential equations of motion in the normal case}

In the normal case $\boldsymbol{B}$ is aligned with the unit vector $\boldsymbol{N}=(0,0, \pm 1)^{T}$. This means that the dipole axis is perpendicular to the orbital plane of the leader, 
Table 1: List of parameters appearing in the dimensional equations of motion.

\begin{tabular}{cl}
\hline Parameter & Physical meaning \\
\hline$\left(\boldsymbol{e}_{r}, \boldsymbol{e}_{n}, \boldsymbol{e}_{t}\right)$ & Unitary vectors defining the local-vertical-local-horizontal \\
& reference system \\
$\boldsymbol{f}_{L}$ & Mean motion of the leader around the Earth \\
$q$ & Lorentz force of the leader acting on the follower, $\boldsymbol{f}_{L}=\left(f_{x}, f_{y}, f_{z}\right)$ \\
$\sigma$ & Sign of the charge $q$ \\
$m$ & Mass of the follower \\
$\boldsymbol{v}_{R}$ & Relative velocity of the follower with respect to the leader \\
$\boldsymbol{B}$ & Magnetic rotating force field created by a dipole \\
$\boldsymbol{N}$ & Unitary vector aligned with $\boldsymbol{B}, \boldsymbol{N}=\left(N_{x}, N_{y}, N_{z}\right)$ \\
$\boldsymbol{\omega}_{c}$ & Angular velocity of the dipole, $\omega_{c}=\left\|\boldsymbol{\omega}_{c}\right\|, \boldsymbol{w}_{c}=w_{c} \boldsymbol{N}$ \\
$\beta$ & Ratio between the motion of the leader $n$ and the angular \\
$B_{0}$ & velocity of the dipole $\omega_{c}, \beta=n / \omega_{c}$ \\
$\mu_{0}$ & Magnetic moment of the dipole created by a coil \\
$n_{c}$ & Vacuum permeability \\
$i_{c}$ & Number of loops of the coil \\
$r_{c}$ & Radrent intensity in the coil \\
$\alpha$ & Unit of length used to define the non-dimensional coordinates \\
\hline
\end{tabular}

pointing to the positive or negative $z$-axis direction according to the sign of \pm 1 . In this case we have,

$$
\boldsymbol{f}_{L}= \pm \frac{q}{m} \frac{B_{0}}{r^{5}}\left(\begin{array}{c}
-\left(x^{2}+y^{2}-2 z^{2}\right) \dot{y}-3 y z \dot{z} \pm \omega_{c} x\left(x^{2}+y^{2}-2 z^{2}\right) \\
\left(x^{2}+y^{2}-2 z^{2}\right) \dot{x}+3 x z \dot{z} \pm \omega_{c} y\left(x^{2}+y^{2}-2 z^{2}\right) \\
3 z\left(y \dot{x}-x \dot{y} \pm \omega_{c}\left(x^{2}+y^{2}\right)\right)
\end{array}\right)
$$

In the analysis we consider only the case $N=(0,0,+1)^{T}$, since the results for the other case can be easily obtained from this one. Using the non-dimensional units, and defining $X=x / \alpha, Y=y / \alpha$, and $Z=z / \alpha$, the equations of relative 
motion for the follower can be written as,

$$
\begin{aligned}
X^{\prime \prime}-2 Y^{\prime}-3 X & =\sigma \frac{-\beta\left(X^{2}+Y^{2}-2 Z^{2}\right) Y^{\prime}-3 \beta Y Z Z^{\prime}+X\left(X^{2}+Y^{2}-2 Z^{2}\right)}{R^{5}}, \\
Y^{\prime \prime}+2 X^{\prime} & =\sigma \frac{\beta\left(X^{2}+Y^{2}-2 Z^{2}\right) X^{\prime}+3 \beta X Z Z^{\prime}+Y\left(X^{2}+Y^{2}-2 Z^{2}\right)}{R^{5}}, \\
Z^{\prime \prime}+Z & =\sigma \frac{3 Z\left[\beta Y X^{\prime}-\beta X Y^{\prime}+\left(X^{2}+Y^{2}\right)\right]}{R^{5}}
\end{aligned}
$$

where $\sigma$ is the sign of the charge $q$, and $R=\sqrt{X^{2}+Y^{2}+Z^{2}}$. It must be noted that, aside from $\sigma$, the only parameter that appears in the above equations is the angular quotient $\beta=n / \omega_{c}$.

The above system admits a first integral, to which we will refer as energy, that is given by,

$$
H_{N}=3 X^{2}-Z^{2}-\sigma \frac{2\left(X^{2}+Y^{2}\right)}{R^{3}}-\left(X^{\prime 2}+Y^{\prime 2}+Z^{\prime 2}\right) .
$$

Next, we consider the symmetries of the model given by the transformations leaving invariant the differential equations of motion. The general pattern of the transformations considered is,

$$
(t, x, y, z) \quad \longrightarrow \quad(D t, A x, B y, C z)
$$

The transformation must be such that if $(x(t), y(t), z(t))$ is a solution of (8), then $(A x(D t), B y(D t), C z(D t))$ must be also a solution. Table 2 lists the seven sets of values of the parameters $A, B, C$ and $D$ fulfilling the above invariance condition. Each set of values has a symmetry element associated, and we have assigned a label to it, both are given in the table.

The time reversing symmetries $(D=-1)$ will be used for the computation of symmetric periodic orbits. Once one of these periodic orbits is computed, using any of the three symmetries preserving time $(D=1)$ we obtain three periodic orbits with the same period $\left(\mathrm{III}_{N}, \mathrm{IV}_{N}, \mathrm{~V}_{N}\right)$. Some of them are potential candidates to be used as nominal orbits for formation flying.

\subsection{Differential equations of motion in the radial case}

In the radial case $\boldsymbol{B}$ is aligned with the radius vector of the leader $\boldsymbol{r}$, so $\boldsymbol{N}=( \pm 1,0,0)^{T}$. Again we only consider $\boldsymbol{N}=(+1,0,0)^{T}$ and using the 
Table 2: Values of the four parameters of the transformation $(t, x, y, z) \longrightarrow(D t, A x, B y, C z)$ that leave invariant the normal differential equations 8 .

\begin{tabular}{cccc}
\hline Model case & Label & $A, B, C, D$ & Symmetry element \\
\hline Normal & $\mathrm{I}_{N}$ & $1,-1,-1,-1$ & $X$ axis \\
& $\mathrm{II}_{N}$ & $-1, \quad 1,-1,-1$ & $Y$ axis \\
& $\mathrm{III}_{N}$ & $-1,-1, \quad 1, \quad 1$ & $Z$ axis \\
& $\mathrm{IV}_{N}$ & $-1,-1,-1, \quad 1$ & Origin \\
& $\mathrm{V}_{N}$ & $1, \quad 1,-1, \quad 1$ & $X-Y$ plane \\
& $\mathrm{VI}_{N}$ & $-1, \quad 1, \quad 1,-1$ & $Y-Z$ plane \\
& $\mathrm{VII}_{N}$ & $1,-1,1,-1$ & $X-Z$ plane \\
\hline
\end{tabular}

previous notation, the equations of relative motion in non-dimensional units can be written as,

$$
\begin{aligned}
X^{\prime \prime}-2 Y^{\prime}-3 X & =\sigma \frac{3 X\left[\beta Z Y^{\prime}-\beta Y Z^{\prime}+\left(Y^{2}+Z^{2}\right)\right]}{R^{5}}, \\
Y^{\prime \prime}+2 X^{\prime} & =\sigma \frac{-\beta\left(Y^{2}+Z^{2}-2 X^{2}\right) Z^{\prime}-3 \beta X Z X^{\prime}+Y\left(Y^{2}+Z^{2}-2 X^{2}\right)}{R^{5}}, \\
Z^{\prime \prime}+Z & =\sigma \frac{-\beta\left(Y^{2}+Z^{2}-2 X^{2}\right) Y^{\prime}+3 \beta X Y X^{\prime}+Z\left(Y^{2}+Z^{2}-2 X^{2}\right)}{R^{5}} .
\end{aligned}
$$

The energy integral is now defined by,

$$
H_{R}=3 X^{2}-Z^{2}-\sigma \frac{2\left(Y^{2}+Z^{2}\right)}{R^{3}}-\left(X^{\prime 2}+Y^{\prime 2}+Z^{\prime 2}\right) .
$$

The parameters of the transformation (10) leaving invariant the differential equations (11), together with the symmetry element and label, are given in Table 3 .

\subsection{Differential equations of motion in the tangential case}

In the tangential case $\boldsymbol{N}=(0, \pm 1,0)^{T}$. As in the other two cases we only consider $\boldsymbol{N}=(0,1,0)^{T}$ and the non-dimensional equations of relative motion 
Table 3: Values of the parameters of the transformation $(t, x, y, z) \longrightarrow(D t, A x, B y, C z)$ that leave invariant the radial differential equations 11 .

\begin{tabular}{cccc}
\hline Model case & Label & $A, B, C, D$ & Symmetry element \\
\hline Radial & $\mathrm{I}_{R}$ & $1,-1,1,-1$ & $X-Z$ plane \\
& $\mathrm{II}_{R}$ & $-1,1,-1,-1$ & $Y$ axis \\
& $\mathrm{III}_{R}$ & $-1,-1,-1,1$ & Origin \\
\hline
\end{tabular}

are given by,

$$
\begin{aligned}
X^{\prime \prime}-2 Y^{\prime}-3 X & =\sigma \frac{\beta\left(X^{2}+Z^{2}-2 Y^{2}\right) Z^{\prime}+3 \beta Y Z Y^{\prime}+X\left(X^{2}+Z^{2}-2 Y^{2}\right)}{R^{5}}, \\
Y^{\prime \prime}+2 X^{\prime} & =\sigma \frac{3 Y\left[\beta X Z^{\prime}-\beta Z X^{\prime}+\left(X^{2}+Z^{2}\right)\right]}{R^{5}}, \\
Z^{\prime \prime}+Z & =\sigma \frac{-\beta\left(X^{2}+Z^{2}-2 Y^{2}\right) X^{\prime}-3 \beta X Y Y^{\prime}+Z\left(X^{2}+Z^{2}-2 Y^{2}\right)}{R^{5}}
\end{aligned}
$$

with the associated energy first integral,

$$
H_{T}=3 X^{2}-Z^{2}-\sigma \frac{2\left(X^{2}+Z^{2}\right)}{R^{3}}-\left(X^{\prime 2}+Y^{\prime 2}+Z^{\prime 2}\right) .
$$

The parameters of the transformation $(10)$ leaving invariant the differential equations (13), together with the symmetry element and label, are given in Table 4

Table 4: Values of the parameters of the transformation $(t, x, y, z) \longrightarrow(D t, A x, B y, C z)$ that leave invariant the tangential differential equations $[13$.

\begin{tabular}{cccc}
\hline Model case & Label & $A, B, C, D$ & Symmetry element \\
\hline Tangential & $\mathrm{I}_{T}$ & $-1,1,1,-1$ & $Y-Z$ plane \\
& $\mathrm{III}_{T}$ & $1,-1,-1,-1$ & $X$ axis \\
& $\mathrm{III}_{T}$ & $-1,-1,-1, \quad 1$ & Origin \\
\hline
\end{tabular}




\section{The normal case. Equilibrium points, zero velocity surfaces, peri- odic and quasi-periodic orbits}

\subsection{Equilibrium points}

Setting $X^{\prime}=Y^{\prime}=Z^{\prime}=0$ and $X^{\prime \prime}=Y^{\prime \prime}=Z^{\prime \prime}=0$ in the equations of motion (8), and solving the associated non-linear system, we obtain the location of the equilibria, that only depend on the sign $\sigma$ of the charge $q$ of the follower. Table 5 gives the results obtained, together with the value of the first integral $H$ at each equilibrium point.

For a positively charged spacecraft $(\sigma=+1)$, there are four equilibria in the $Y-Z$ plane, with label $1_{N}$, and four in the $X-Z$ plane, with label $2_{N}$. When $\sigma=-1$ there are only two equilibria, both on the $X-$ axis, with label $3_{N}$.

Table 5: Location of the equilibrium points of system (8) and value $H$ of the Hamiltonian (9) as a function of the sign $\sigma$ of the charge $q$.

\begin{tabular}{cccccc}
\hline Label & $\sigma$ & $X$ & $Y$ & $Z$ & $H$ \\
\hline $1_{N}$ & +1 & 0 & $\pm \sqrt{2} Z$ & $\pm\left(\frac{2}{3 \sqrt{3}}\right)^{1 / 3}$ & -1.587401 \\
$2_{N}$ & +1 & $\pm\left(\frac{1}{12 \sqrt{6}}\right)^{1 / 3}$ & 0 & $\pm \sqrt{5} X$ & -0.629960 \\
$3_{N}$ & -1 & $\pm\left(\frac{1}{3}\right)^{1 / 3}$ & 0 & 0 & 4.326748 \\
\hline
\end{tabular}

The linear stability of the equilibrium points depends on the eigenvalues of the Jacobian matrix of the differential equations at the equilibrium point. Due to the symmetry of the system, the characteristic polynomial $p(\lambda)$ of the Jacobian has only odd-order terms, so it can be written as

$$
p(\lambda)=\lambda^{6}+b \lambda^{4}+c \lambda^{2}+d,
$$

or, denoting by $\kappa=\lambda^{2}$, as the cubic polynomial

$$
q(\kappa)=\kappa^{3}+b \kappa^{2}+c \kappa+d .
$$

The coefficients $b, c$ and $d$ in the above polynomials depend on the equilibrium point and the parameter $\beta$ (see [18]). The discriminant of $q(\kappa)=0$ is given by

$$
\Delta=b^{2} c^{2}-4 b^{3} d-4 c^{3}+18 b c d-27 d^{2} .
$$


The roots of $q(\kappa)=0$ can be classified according to the value of $\Delta$. If $\Delta>0$, 16. has three distinct real roots, if $\Delta=0$, it has a real root with multiplicity three, while if $\Delta<0$ it has one real root and two complex conjugates ones. Note that the eigenvalues of the Jacobian, which determine the stability of the equilibrium point, come in pairs: i.e., for real ones we have $\pm \lambda$ with $\lambda \in \mathbb{R}$, while for complex ones we have the conjugated pair $\lambda, \bar{\lambda}$ with $\lambda \in \mathbb{C}$.

In the case under consideration, the characteristic polynomials of the three different kinds of equilibrium points are:

$$
\begin{array}{ll}
1_{N}: & p(\lambda)=\lambda^{6}+\left(\frac{\beta^{2}}{2}+2\right) \lambda^{4}+\frac{-\beta^{2}+8 \beta+5}{3} \lambda^{2}+6, \\
2_{N}: & p(\lambda)=\lambda^{6}+\left(14 \beta^{2}+12 \beta+8\right) \lambda^{4}+\frac{-40 \beta^{2}+80 \beta-1}{3} \lambda^{2}-60, \\
3_{N}: & p(\lambda)=\lambda^{6}+\left(9 \beta^{2}+12 \beta+8\right) \lambda^{4}+\left(90 \beta^{2}+120 \beta-47\right) \lambda^{2}-270 .
\end{array}
$$

Clearly, all the characteristic polynomials depend on the value of the angular velicities quotient $\beta=n / \omega_{c}$. According to this, we have explored the evolution of the eigenvalues of the Jacobian as a function of $\beta$. We have assumed that the rate of rotation of the dipole $\omega_{c}$ is such that only relative small values need to be considered. Here we present the resuts for $\beta \in[-10,10]$, except for the equilibrium point $1_{N}$ for which the interval has been enlarged up to a maximum value of $\beta=25$.

Fig. 2 shows the real and imaginary parts of the six eigenvalues of the equilibrium points $1_{N}, 2_{N}$ and $3_{N}$ as a function of the parameter $\beta$.

The four equilibria with label $1_{N}$ are either of saddle $\times$ saddle $\times$ centre type or complex saddle $\times$ centre type, which means that, according to Lyapunov center theorem, there is always a one-parameter family of periodic orbits around each of the four equilibrium points. The equilibria with index $2_{N}$, and $3_{N}$ are of saddle $\times$ centre $\times$ centre type; the dimension of their associated stable and unstable manifolds is one, while the one of the centre manifold is four. For these points there are two families of periodic orbits associated. It is also observed that one pair of pure imaginary eigenvalues remain constant for $3_{N}$, see Fig. 2 175 (right). 

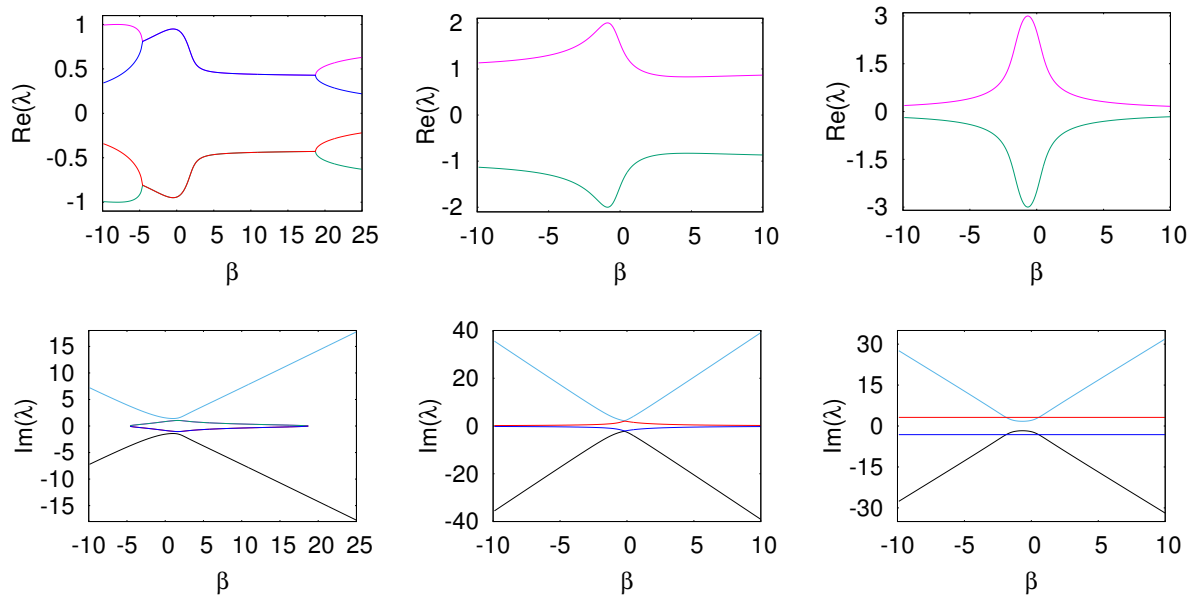

Figure 2: Behaviour of the eigenvalues associated to the equilibrium points $1_{N}$ (left), $2_{N}$ (middle) and $3_{N}$ (right) as a function of $\beta$. Only the non-zero values of the real and imaginary parts of the eigenvalues are plotted. The real and the imaginary part of the same eigenvalue are in same color: $\lambda_{1}$ in magenta, $\lambda_{2}$ in green, $\lambda_{3}$ in light blue, $\lambda_{4}$ in black, $\lambda_{5}$ in red, and $\lambda_{6}$ in blue.

\subsection{Zero velocity surfaces}

The zero velocity surfaces (ZVS) are defined, by means of the energy function (9), as the set of points $(X, Y, Z)$ in the configuration space such that

$$
X^{\prime 2}+Y^{\prime 2}+Z^{\prime 2}=F(X, Y, Z)-H=0 .
$$

They separate the configuration space in different components determining the forbidden and allowable regions of motion. Because of this separation property, zero velocity curves and surfaces have been used a lot in many problems; see, for instance, [4, 6] for some Celestial Mechanics applications). We will focus on energy levels around the ones of the equilibrium points, since the topology structure of ZVS changes when the energy goes through these values.

Fig. 3 shows the behaviour of the ZVS for values of the energy $H$ close to $H_{1 N}=-1.587401, H_{2 N}=-0.629960$, and $H_{3 N}=4.326748$.

In any of the regions of the configuration space $(X, Y, Z)$ determined by the ZVS, the follower can only move inside certain closed regions or unbounded 

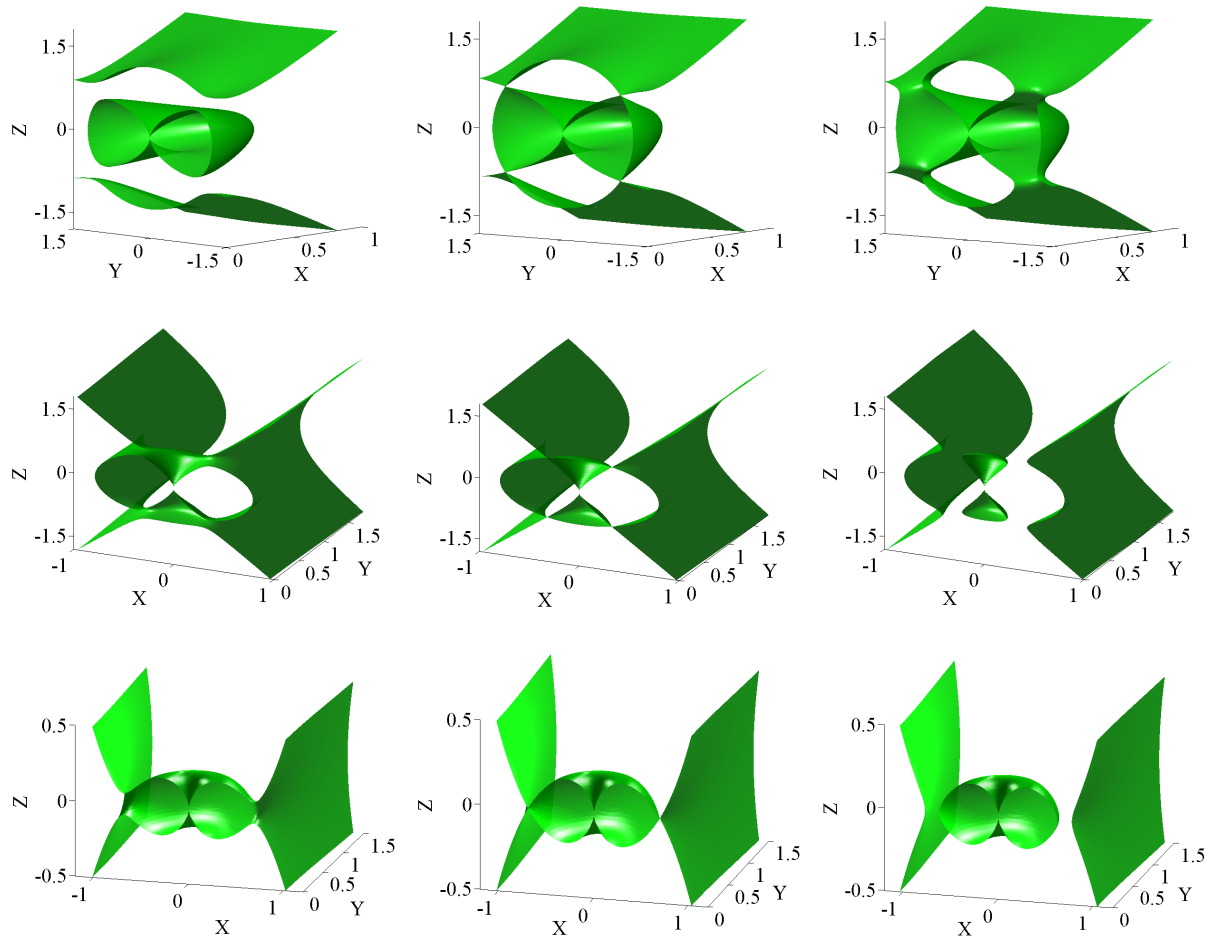

Figure 3: Evolution of ZVS near the equilibrium points $1_{N}, \sigma=+1$ (top), $2_{N}, \sigma=+1$ (middle) and $3_{N}, \sigma=-1$ (bottom), as the energy level varies around its value of at the equilibrium point $H_{E P}$. From left to right, the values of $H$ are $H_{E P}-0.05, H_{E P}$, and $H_{E P}+0.05$, respectively. The surfaces are represented up to their intersection with a symmetry plane: $X=0$ (top row), and $Y=0$ (two bottom rows).

ones. For the equilibrim point with label $1_{N}$, there are three unconnected components of motion when $H_{1 N}<-1.587401$. These three components met at the equilibrium points when $H_{1 N}=-1.587401$ and when $H_{1 N}>-1.587401$ there is only one connected component, where the motion can take place (the spacecraft is free to move in the whole configuration space connected through the 'bottle-necks' passages around the four equilibria).

For the equilibrim point with label $2_{N}$, there is only one connected component of motion when $H<H_{2 N}$, when $H>H_{2 N}$, the admissible region of motion has four components, two of them are closed and near the origin (above 
and below the $(X-Y)$ plane), and the other two are unbounded (to the right and to the left of the two hyperbolic surfaces). These four regions have a contact at the equilibrium points when $H_{2 N}=-0.629960$.

The behaviour of the equilibrim point with label $3_{N}$ close to $H_{3 N}=4.326748$

\subsection{Some families of periodic orbits}

In this section we show the numerical results obtained in the computation of the families of periodic, and quasi-periodic orbits (tori) in the central manifolds associated to the equilibrium points.

The computation of the families of periodic orbits has been done using the arc-parameter in the continuation method, following section 7.1 . The symmetries of the differential equations (8), previously discussed in Section 2.1, have been used for the computation of different families of symmetric periodic orbits.

Due to the Hamiltonian character of the system under consideration, there are three couples of characteristic multipliers (eigenvalues of the monodromy matrix) associated to any periodic orbit: $\left(\lambda_{1}, \lambda_{1}^{-1}\right),\left(\lambda_{2}, \lambda_{2}^{-1}\right)$ and $\left(\lambda_{3}=\lambda_{3}^{-1}=\right.$ 1) that determine their stability properties. According to their distribution in the complex plane we have introduced the following four configurations: $B_{1}, B_{2}$, $B_{3}$ and $B_{4}$, see Figure 4. The non-generic cases, with multiplier +1 with multiplicity 4 and 6 , are not considered. The first configuration $B_{1}$ corresponds to a real saddle, $B_{2}$ to a central saddle, $B_{3}$ to a totally elliptic centre (stable periodic orbit), and $B_{4}$ to a complex saddle with multiples as complex quadrupole. When there is a transition between two of these different configurations bifurcations may occur [25].

The isoenergetic families of 2D tori have been computed keeping the energy $H$ fixed and varying the rotation number and the return time $(\rho, T)$ during the continuation, according to section 7.2 . 


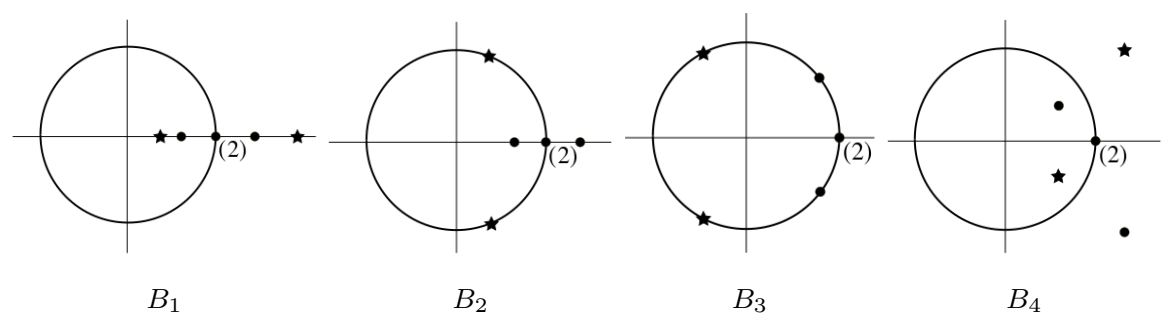

Figure 4: The four generic configurations of the characteristic exponents around the unit circle. The stars correspond to $\left(\lambda_{1}, \lambda_{1}^{-1}\right)$, the dots to $\left(\lambda_{2}, \lambda_{2}^{-1}\right)$, and " $(2)$ " indicates the multiplicity of $\lambda_{3}$.

\subsubsection{Periodic orbits around the $1_{N}$ equilibria}

There are four equilibrium points of type $1_{N}$ located at $\left(0, \pm \sqrt{2}(2 /(3 \sqrt{3}))^{\frac{1}{3}}\right.$, $\left.\left.{ }_{225} \pm(2 /(3 \sqrt{3}))^{\frac{1}{3}}\right)\right)$, all of them with one one-parameter family of periodic orbits associated. We will focus in the two equilibria with $Z>0$, that are symmetric w.r.t the $Y=0$ plane. Since both points are in the $Y-Z$ plane, for the computation of symmetric periodic orbits around them we have used the symmetry, labelled as $\mathrm{VI}_{N}$, about this plane. Note that only one of these families has to be computed, as the families around the other equilibrium points are just symmetric images with respect to the $Z=0$ plane.

For the computations that follow we have used as reference values for the parameter $\beta$ the values 2 and -2 , as they are qualitatively equivalent to $\beta>0$ and $\beta<0$, respectively.

For $\beta=2$ the associated eigenvalues to the equilibrium points are $( \pm 0.6509 \pm$ $1.0291 i, \pm 1.6520 i)$ and if $\boldsymbol{v}^{c}=\boldsymbol{v}_{r}^{c} \pm \boldsymbol{v}_{i}^{c}$ denotes the eigenvector associated to the purely imaginary eigenvalue, $\pm 1.6520 i$, we have

$\boldsymbol{v}_{r}^{c}=[0.2477,0,0,-0.1968,0.7250]^{T}, \boldsymbol{v}_{i}^{c}=[0,0.1191,-0.4389,0.4092,0,0]^{T}$.

This data has been used to determine the first orbit of the family of periodic around the equilibrium point with $\left.Y>0:\left(0,+\sqrt{2}(2 /(3 \sqrt{3}))^{\frac{1}{3}},(2 /(3 \sqrt{3}))^{\frac{1}{3}}\right)\right)$. The family ends at the point $\left.\left(0,-\sqrt{2}(2 /(3 \sqrt{3}))^{\frac{1}{3}},(2 /(3 \sqrt{3}))^{\frac{1}{3}}\right)\right)$, which is the symmetric of the initial one w.r.t the $Y=0$ plane. This is, the families ema- 
nating from both points are connected at any intermediate orbit of them, for

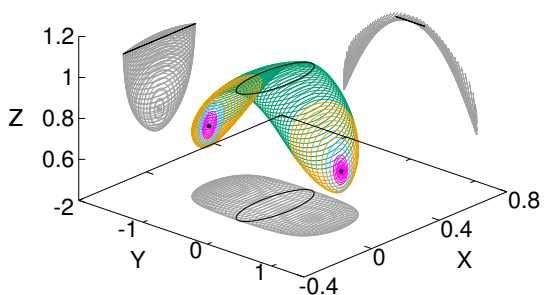

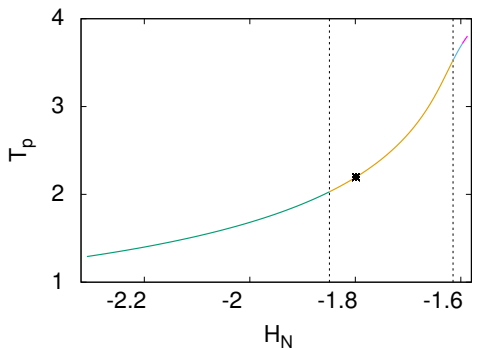

Figure 5: For $\beta=2,3 \mathrm{D}$ representation and 2D coordinate projections of the periodic orbits of the two connected families around the $1_{N}$ equilibrium points $\left.\left(0, \pm \sqrt{2}(2 /(3 \sqrt{3}))^{\frac{1}{3}},(2 /(3 \sqrt{3}))^{\frac{1}{3}}\right)\right)$ (left). The right had side plot displays the energy $H$ vs. period $T$ characteristic curve of the family starting at the equilibrium point and finishing at the periodic orbit represented in black. The type of orbits follows the transtion: $B_{4}$ (magenta) $\rightarrow B_{1}$ (blue) $\rightarrow B_{2}$ (orange) $\rightarrow B_{3}$ (green). The vertical lines indicate the bifurcation points.

Fig. 5 also shows the characteristic curve (energy $H$ vs. period $T$ ) and the results of the stability analysis. Different colors denote different configurations of the multipliers of the periodic orbits. Two period-doubling bifurcations are detected along the family when one pair of multipliers cross -1 . They are indicated by two vertical lines.

The family starts at the right point of the characteristic curve, with $H_{N}=$ -1.587402 . The period of this orbit is very close to $(2 \pi i) / \lambda=(2 \pi) / 1.6520$ which, according to Lyapunov theorem, is the limit value of the periods of the family. The first orbits of the family are of type $B_{4}$, according to the clasification previously introduced. The family transits to type $B_{1}$, when the energy goes through $H_{N}=-1.597089$, and to type $B_{2}$ (as a real saddle) at 
$H_{N}=-1.614741$, where a period-doubling bifurcation occurs. The family is unstable up to $H_{N}=-1.854221$, where a second period-doubling bifurcation is detected, afterwards the family is of type $B_{3}$ (green) and becomes stable. The black point in the characteristic curve indicates the periodic orbit from which later we compute an iso-energetic family of $2 \mathrm{D}$ tori (section 3.4.

Due to the type $\mathrm{VII}_{N}$ symmetry, the orbits to the left of the "connecting" (black) orbit -left branch- are the images of the orbits to the right of this orbit right branch-. As a consequence, the characteristic curve of both branches is the same. The periodic orbit connecting the two branches corresponds to the most left point of the characteristic curve, and the associated multipliers correspond to a degenerated centre with four multipliers equal to +1 . Moreover, the middle (black) periodic orbit is symmetric with respect to both the $X-Z$ plane and the $Y-Z$ plane, and is "almost" parallel to the $X-Y$ plane; its maximum displacement along the $Z$ direction is of the order of $1 \times 10^{-5}$ non-dimensional units. These "almost" parallel orbits could be used as potential nominal orbits for a displaced observation mission.
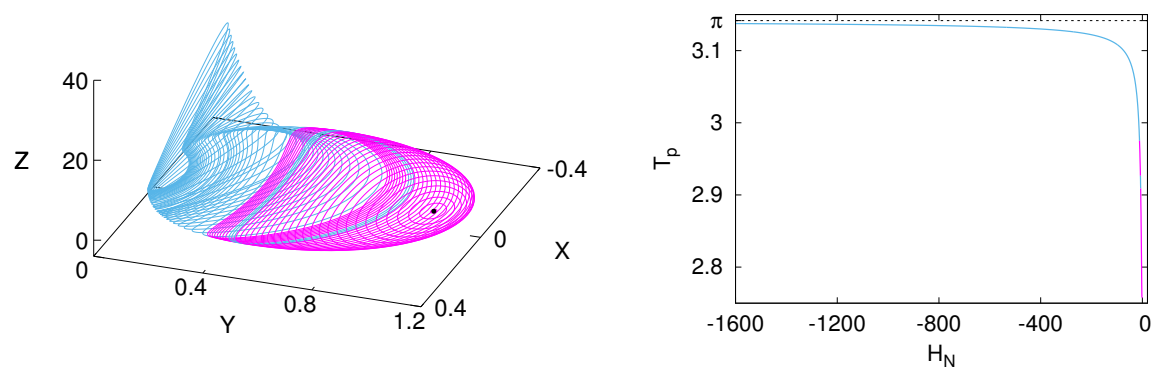

Figure 6: For $\beta=-2$, 3D representation of the periodic orbits of the family around the $1_{N}$ equilibrium point $\left.\left(0, \sqrt{2}(2 /(3 \sqrt{3}))^{\frac{1}{3}},(2 /(3 \sqrt{3}))^{\frac{1}{3}}\right)\right)$ (left). The right had side figure displays the energy $H$ vs. period $T$ characteristic curve of the family starting at the equilibrium point. The colors in both figures indicate different configurations of the multipliers. Their types go through the following transitions: $B_{4}$ (magenta) to $B_{1}$ (blue), back to $B_{4}$ (magenta) and $B_{1}$ (blue).

Fig. 6 shows, for $\beta=-2$, the behaviour of the orbits of the family associated to the same as before equilibrium point (with $Y>0$ ) together with its character- 
istic curve. The continuation of this family has been stopped when $|Z|>45$. It can be seen that, as the energy decreases, the orbits become very large along the $Z$ direction, and their period tends to a value close to $\pi$ (we have not found an explanation of this fact). Now, the connection of both families has disappeared, and one family is symmetric to the other without common orbits. According to the behaviour of the characteristic multipliers, the orbits are always unstable, although their type follows the transitions: $B_{4} \rightarrow B_{1} \rightarrow B_{4} \rightarrow B_{1}$.
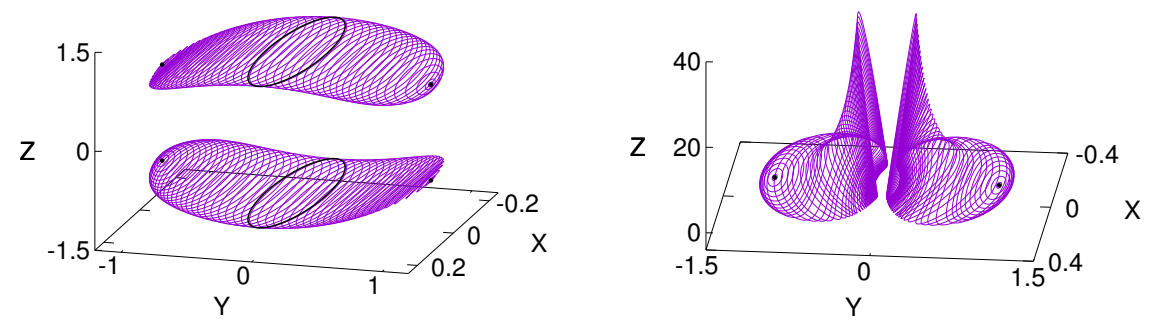

Figure 7: 3D representation of the families of periodic orbits around the equilibrium points of type $1_{N}$. The left hand side plot shows the four families corresponding to $\beta=2$. The right hand side shows two of the four families with $Z>0$ for $\beta=-2$ (the other two families are symmetric w.r.t the $Z=0$ plane).

All four families emanating from the $1_{N}$ equilibrium points, for $\beta=2$ and for $\beta=-2$, are shown together in Fig. 7 .

\subsubsection{Periodic orbits around the $2_{N}$ equilibria} $0, \pm \sqrt{5}\left(1 /(16 \sqrt{6})^{1 / 3}\right)$. As in the preceding case, we mainly consider the point with $X>0$ and $Z>0$. The periodic orbits around the remaining points can be obtained using suitable symmetries. As was mentioned in Sect. 3.1, the centre manifold associated to all these equilibria has dimension four and so it contains with their associated characteristic curves for $\beta=2$. The $\operatorname{VII}_{N}$ symmetry w.r.t. the $X-Z$ plane has been used for their computation.

Analogously to what happens in the $1_{N}$ case when $\beta>0$, one of the fam- 

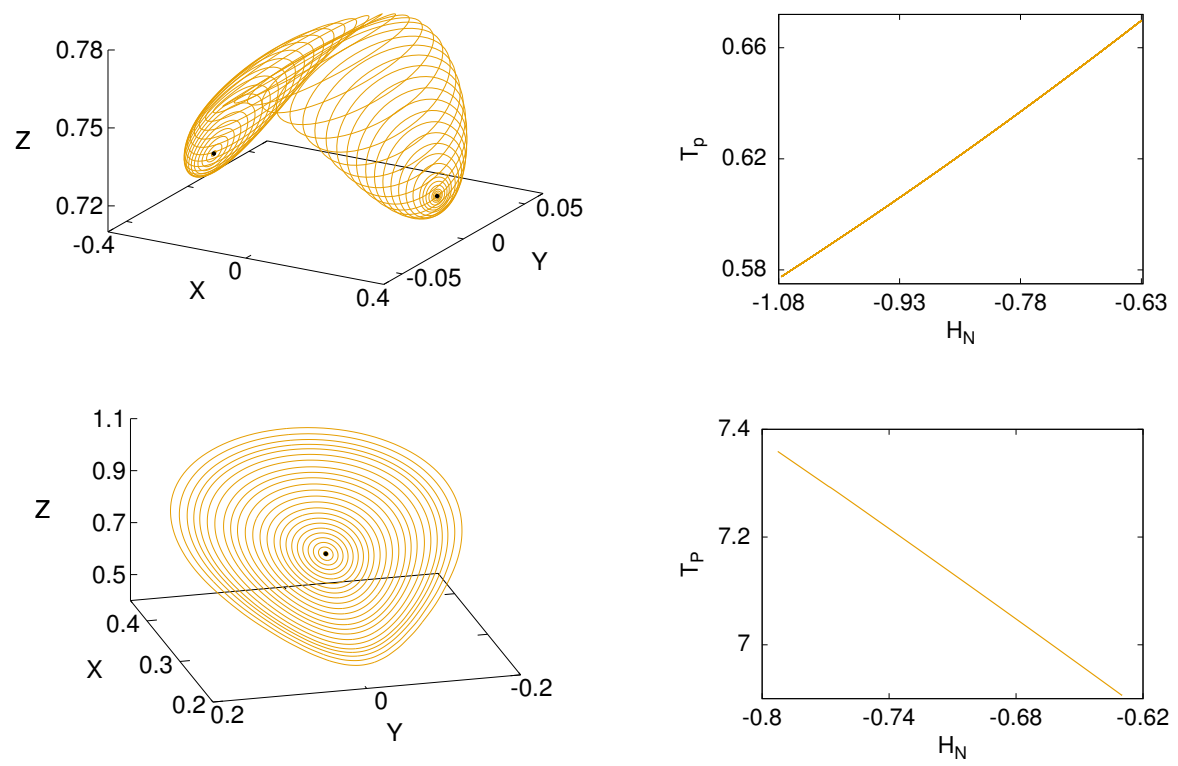

Figure 8: For $\beta=2$, the two families of periodic orbits emanating from the equilibrium point of type $2_{N}$ with $X>0$ and $Z>0$ (left), with the 3D representation of the orbits in the left hand side figures and the associated characteristic curves at the right hand side ones. Both families are unstable and of type $B_{2}$.

ilies (top line of Fig. 8) has its termination at the equilibrium point symmetric with respect to the $X=0$ plane and, in fact, the families around $\left(+\left(1 /(16 \sqrt{6})^{1 / 3}, 0, \pm \sqrt{5}\left(1 /(16 \sqrt{6})^{1 / 3}\right)\right.\right.$ and $\left(-\left(1 /(16 \sqrt{6})^{1 / 3}, 0, \pm \sqrt{5}\left(1 /(16 \sqrt{6})^{1 / 3}\right)\right.\right.$ are the same one. All the periodic orbits around these two points are symmetric w.r.t. the $X-Z$ plane, and the orbits around one point are the symmetric images of the ones around the other point w.r.t. the $X=0$ plane $\left(\mathrm{VI}_{N}\right.$ symmetry). As a consequence, the characteristic curves of both families are the one displayed in the figure but travelled twice. We note that the left point of this curve corresponds to the orbit that connects the two families and its multipliers correspond to the ones of a degenerated centre with the multiplier +1 with multiplicity 4. For the other family, in the bottom line of the figure, the left point of the characteristic curve corresponds to its termination, where the orbit is a degenerated real saddle with the multiplier +1 also with multiplicity 4 . 
The characteristic curves of the two families, shown in Fig. 8, display a monotonous linear dependence between the energy and the period, and all the orbits of both families are unstable of type $B_{2}$ (saddle $\times$ centre).

Along the family displayed in the bottom line of Fig. 8, we have detected six bifurcations when one pair of purely imaginary multipliers become equal to +1 , and so one of the traces is equal to +2 . The same kind of bifurcation appears in the three-dimensional restricted three-body problem (see Ref. [26]). The orbits of the bifurcated families, and the one from which they are born, together with the associated characteristic curves are shown in Fig. 9. All these families are of unstable orbits, with multiplier configuration of type $B_{1}$ (saddle $\times$ saddle) for the third and fifth families while the remaining ones are of type $B_{2}$ (saddle $\times$ centre).
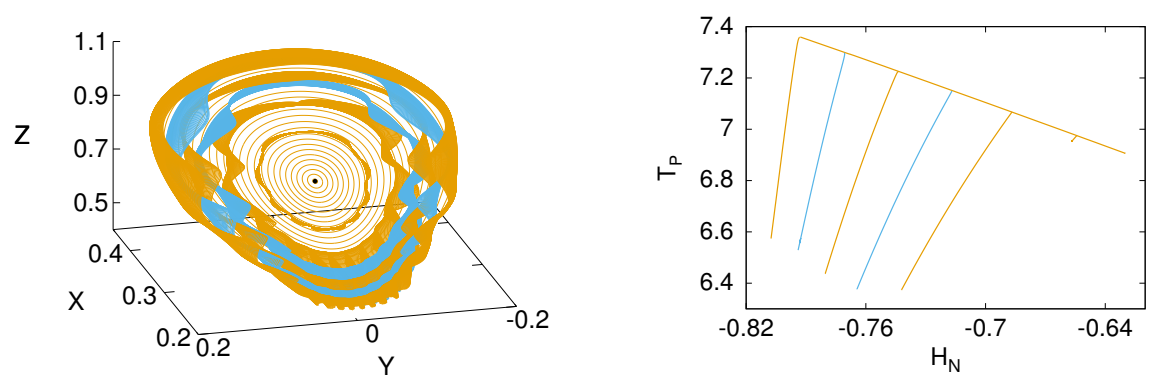

Figure 9: The six bifurcated families of periodic orbits born from bifurcation points when one pair of purely imaginary multipliers become +1 , together with the family from which they emanate.

The periodic orbits associated to the other three equilibria can be obtained using the symmetries $\mathrm{III}_{N}, \mathrm{IV}_{N}$ and $\mathrm{V}_{N}$. They are shown in Fig. 10. We stress that there is no qualitative difference with other values of $\beta$ different from the one shown here.

\subsubsection{Periodic orbits around the $3_{N}$ equilibria}

There are only two equilibrium points of type $3_{N}$, their coordinates are $325\left( \pm(1 / 3)^{1 / 3}, 0,0\right)$. For any value of $\beta$, the Jacobian at these points has four eigen- 


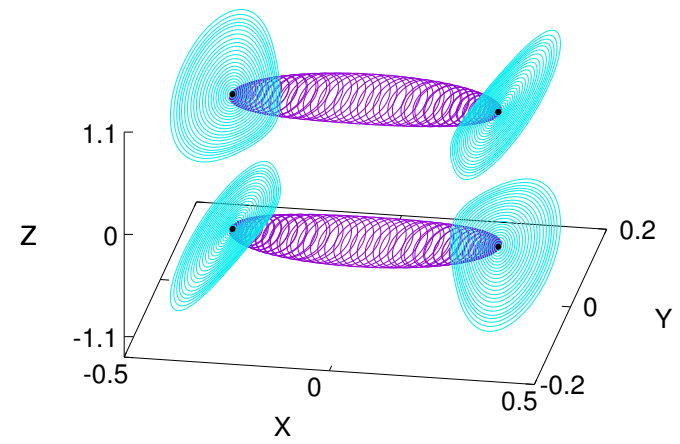

Figure 10: For $\beta=2$, the six families of periodic orbits emerging from the four equilibria of type $2_{N}$, with the families associated to $\lambda_{1}$ in purple and the ones to $\lambda_{2}$ in cyan. The orbits have been represented using the ones computed for $X>0, Z>0$ and the $\operatorname{III}_{N}, \operatorname{IV}_{N}$ and $\mathrm{V}_{N}$ symmetries.

values, $\pm \lambda_{1} i$ and $\pm \lambda_{2} i$, purely imaginary and two, $\pm \lambda_{3}$, real. As a consequence, there are two families of periodic orbits in the centre manifold associated to each point. Taking $\beta=2$ for illustration purposes (again the qualitative behaviour of the periodic orbits is independent of $\beta$ ), we have $\lambda_{1}=7.6460, \lambda_{2}=3.1623$ and $\lambda_{3}=0.6796$, so the periods of the two families tend to $2 \pi / \lambda_{1}=0.821761092$ and $2 \pi / \lambda_{2}=1.986903617$, respectively. Some orbits of both families, together with the characteristic curves, are shown in Fig. 11 .

The orbits of the family associated with $\lambda_{1}=7.6460$ are planar. Close to the equilibrium point the orbits are unstable, at $H_{N}=3.827218$ (the first vertical dashed line) a saddle-node bifurcation is detected and the family transits from type $B_{2}$ (unstable) to $B_{3}$ (stable). As shown in Fig. 11, close to its end the orbits become infinitesimal oscillations around the origin, where the family ends.

The orbits of the family associated to $\lambda_{2}=3.1623$ are 3 -dimensional and reach a planar orbit $(Z=0)$ with $H_{N}=3.868084$, that is marked with an $A$ 340 in the left plots of the two bottom lines of Fig. 11 . The orbits of this piece of the family go from type $B_{2}$ (unstable) to type $B_{3}$ (stable) at $H_{N}=3.569571$, where there is a saddle-node bifurcation. Very close to the end of the family $\left(H_{N}=3.868084\right)$, the type changes from $B_{3}$ to $B_{4}$, and then back to $B_{3}$ through two Neimark-Sacker bifurcations. 

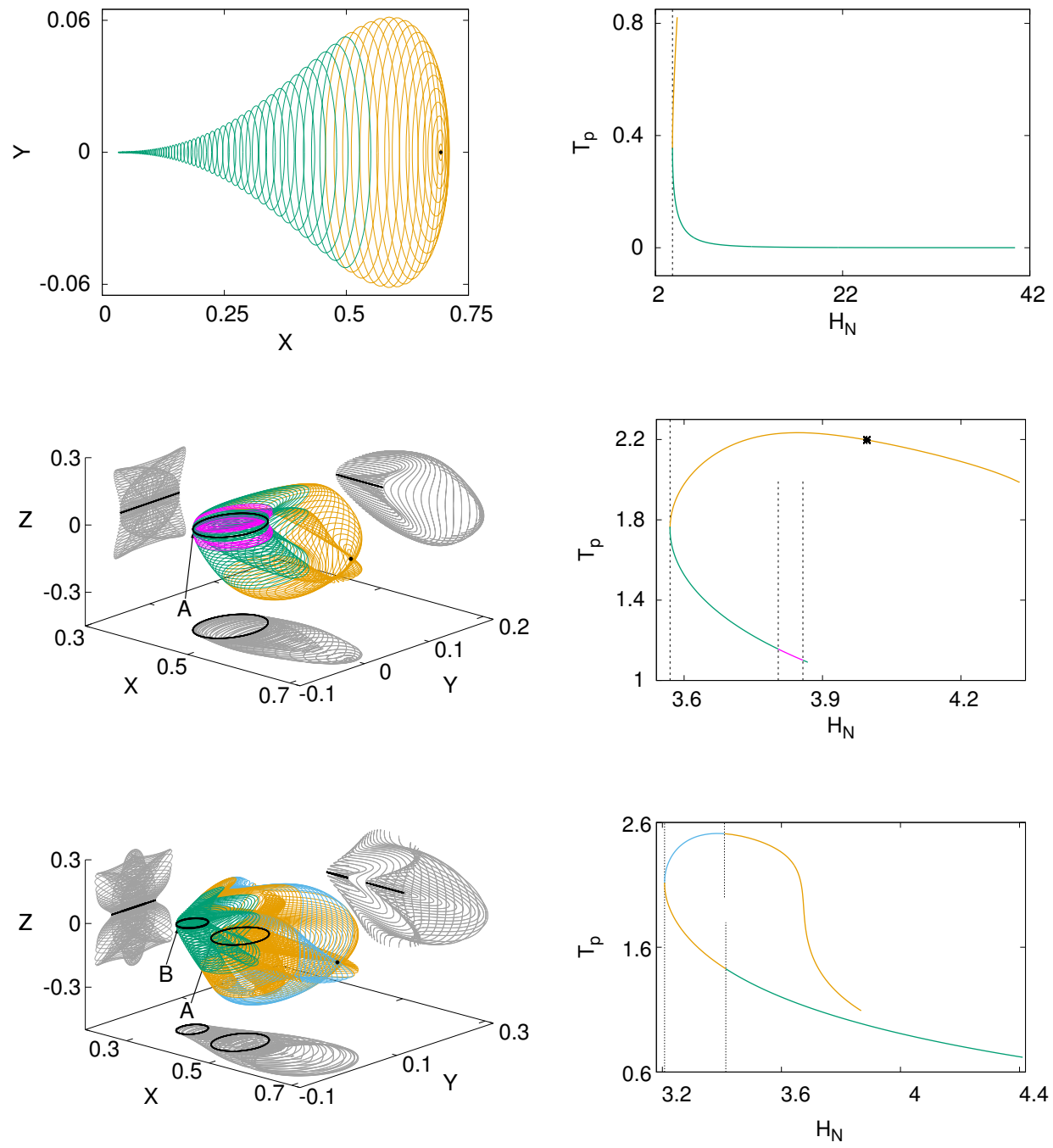

Figure 11: The two families of periodic orbits emanating from the $3_{N}$ equilibrium point with $X>0$. The top row shows some orbits of the family associated with the eigenvalue $\lambda_{1}$ together with the characteristic curve. All orbits of this family are planar; those of type $B_{3}$ are in green (stable orbits), and those of type $B_{2}$ in purple (unstable orbits). The second and third lines show the results for the family associated to the eigenvalue $\lambda_{2}$. The evolution of the family up to the planar orbit with $Z=0$ (label A) is displayed in the middle line, the orbits in green are the stable ones and the colors indicate the type evolution of the orbits, that follows the sequence $B_{2}$ (orange) $\rightarrow B_{3}$ (green) $\rightarrow B_{4}$ (magenta) $\rightarrow B_{3}$ (green). The bottom line shows the orbits obtained from the orbit with label A up to the orbit with label $\mathrm{B}$ with the same color criteria as above. In these last orbits the type transitions follow the sequence: $B_{2}$ (orange) $\rightarrow B_{1}$ (blue) $\rightarrow B_{2}$ (orange) $\rightarrow B_{3}$ (green). 

puted up to values of $\rho$ close to the resonance $\rho=2 \pi \frac{15}{64}$, indicated as dashed 
line in the top right hand side plot of Fig. 12 (characteristic curve). The same figure also shows a 3D representation of some of the computed tori together with the invariant curve used for its computation.
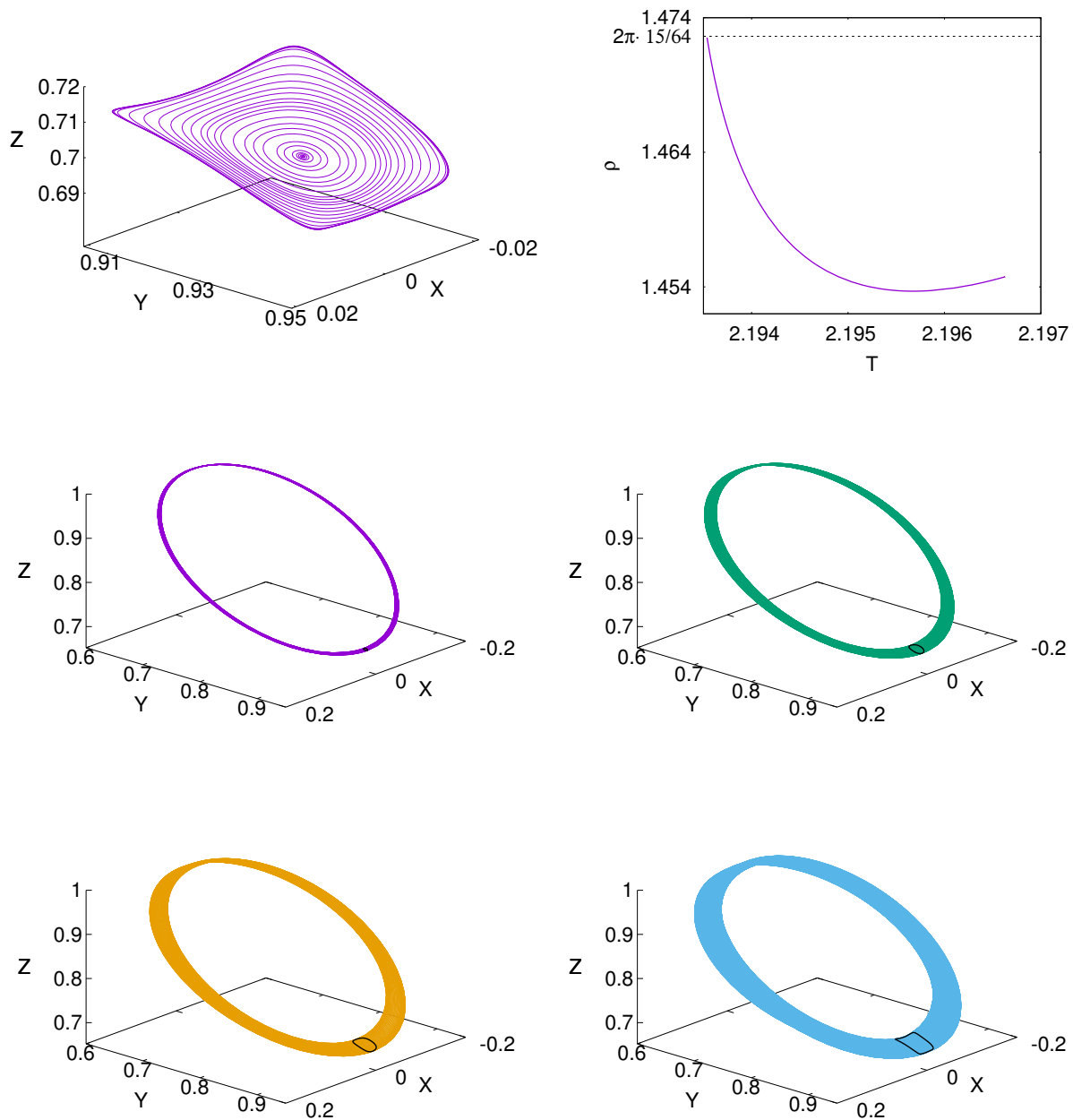

Figure 12: For $H_{N}=-1.798693$, invariant curves $\varphi$ associated to the isoenergetic family of $2 \mathrm{D}$ tori around the periodic orbit with label $1_{N 1}$ (top left plot). The top right plot shows the rotation number $\rho$ in front of return time $T$ of the isoenergetic family of tori. The family has been computed up to values of $\rho$ close to the resonance $15 / 64$. The two bottom rows show some of the tori of the family. In all the plots we have included the invariant curves $\varphi$ used for their computation (in black). 
Fig. 13 shows the results obtained for the isoenergetic family of tori around the periodic orbit with label $3_{N 1}\left(H_{N}=3.996198\right)$. This family has been computed up to the resonance with $\rho=\frac{2 \pi}{29}$. As it is clearly seen looking at the invariant curves, the shape becomes more complex as the tori increase in size and, as a consequence, the number of frequencies $N_{f}$ required for their accurate computation varies along the family. To show this fact we have plotted the invariant curves in different colors according to the values of $N_{f}$. The continuation procedure terminates when we approach the $\frac{1}{29}$ resonance, that corresponds to the maximum allowed value of $N_{f}=128$.

Fig. 14 shows the results obtained for the isoenergetic family of tori around the periodic orbit with label $3_{N 3}\left(H_{N}=4.008587\right)$. Recall that this orbit belongs to the same family as the periodic orbit $3_{N 1}$. This family has been computed up to the resonance corresponding to $\rho=\frac{2 \pi}{27}$, when the number of frequencies required for the computation of the invariant curve $\varphi$ is greater that the maximum allowed value $N_{f}=128$.

\section{The radial case. Equilibrium points, zero velocity surfaces, peri- odic orbits}

\subsection{Equilibrium points}

In the radial case, and for $\sigma=+1$, there are two equilibria on the $Z$-axis, with label $1_{R}$. If $\sigma=-1$, there are four equilibrium points in the $X-Y$ plane with $2_{R}$, and four in the $X-Z$ plane, with $3_{R}$. Table 8 gives the results obtained, together with the value of the first integral $H$ at each equilibrium point.

The characteristic polynomials of the different kinds of equilibrium points 

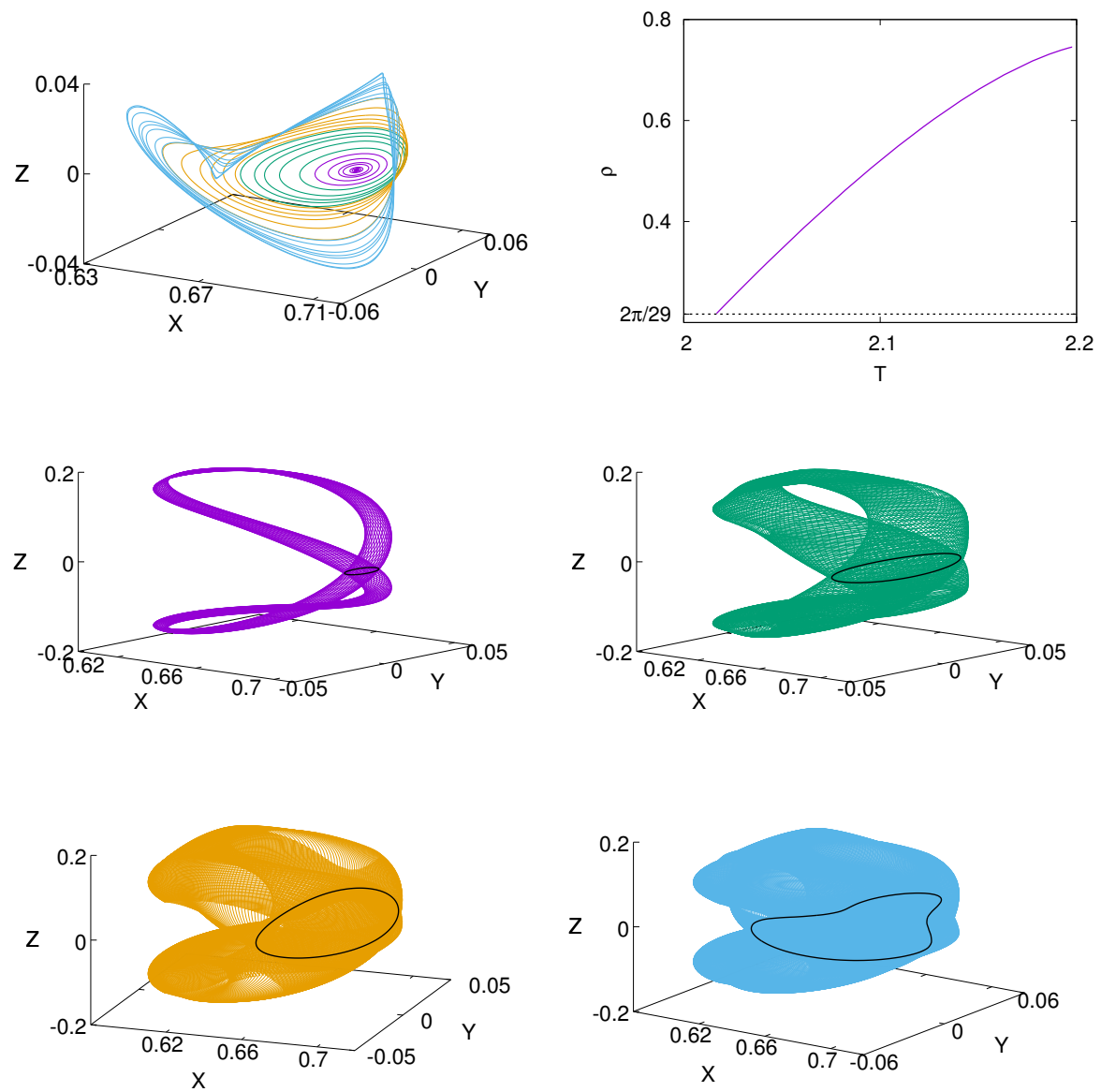

Figure 13: For $H_{N}=3.996198$, invariant curves $\varphi$ associated to the isoenergetic family of 2D tori around the periodic orbit with label $3_{N 1}$ (top left plot). The different colors of the curves correspond to the number of frequencies required for their computation $(\leq 16$ magenta, $\leq 32$ green, $\leq 64$ orange and $\leq 128$ blue).The top right plot shows the rotation number $\rho$ in front of return time $T$ of the isoenergetic family of tori. The family has been computed up to values of $\rho$ close to the resonance $1 / 29$. The two bottom rows show some of the tori of the family. In all plots we have included the invariant curves $\varphi$ used for their computation (in black).

considered are:

$$
\begin{aligned}
1_{R}: & p(\lambda) & =\lambda^{6}+\beta^{2} \lambda^{4}-\left(6 \beta^{2}+3\right) \lambda^{2}+18, \\
2_{R}: & p(\lambda) & =\lambda^{6}+\left(\frac{9}{2} \beta^{2}+2\right) \lambda^{4}+\left(9 \beta^{2}-17\right) \lambda^{2}-18, \\
3_{R}(Z=\sqrt{X}): & p(\lambda) & =\lambda^{6}+\left(10 \beta^{2}-12 \beta\right) \lambda^{4}+\left(24 \beta^{2}-48 \beta-15\right) \lambda^{2}+36, \\
3_{R}(Z=-\sqrt{X}): & p(\lambda) & =\lambda^{6}+\left(10 \beta^{2}+12 \beta\right) \lambda^{4}+\left(24 \beta^{2}+48 \beta-15\right) \lambda^{2}+36,
\end{aligned}
$$



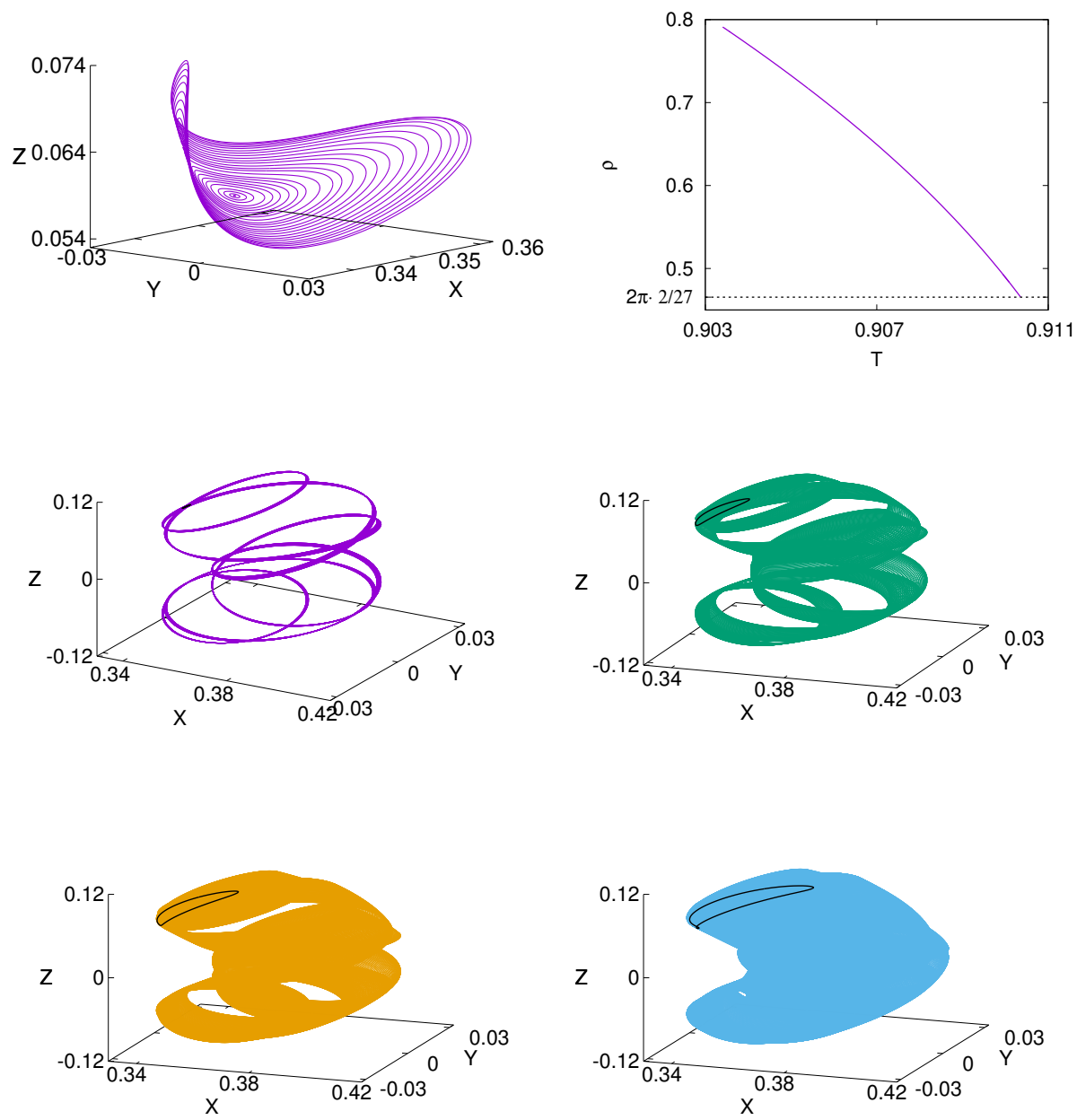

Figure 14: For $H_{N}=4.008587$, invariant curves associated to the isoenergetic family of $2 \mathrm{D}$ tori around the periodic orbit with label $3_{N 2}$ (top left plot). The top right plot shows the rotation number $\rho$ in front of return time $T$ of the isoenergetic family of tori. The family has been computed up to values of $\rho$ close to the resonance $2 / 27$. The two bottom rows show some of the tori of the family. In all plots we have included the invariant curves $\varphi$ used for their computation (in black).

We have explored the evolution of the eigenvalues of the Jacobian as a function of $\beta$ which has been considered between $[-10,10]$. Fig. 15 shows the real 
Table 7: Location of the equilibrium points of system 11 and value $H$ of the Hamiltonian 12 as a function of the sign $\sigma$ of the charge $q$.

\begin{tabular}{cccccc}
\hline Label & $\sigma$ & $X$ & $Y$ & $Z$ & $H$ \\
\hline $1_{R}$ & +1 & 0 & 0 & \pm 1 & -3 \\
$2_{R}$ & -1 & $\pm\left(\frac{2}{9 \sqrt{3}}\right)^{1 / 3}$ & $\pm \sqrt{2} X$ & 0 & 2.289428 \\
$3_{R}$ & -1 & $\pm\left(\frac{1}{4 \sqrt{2}}\right)^{1 / 3}$ & 0 & $\pm \sqrt{X}$ & 1.88988 \\
\hline
\end{tabular}

and imaginary parts of the six eigenvalues of the equilibrium points as a function of the parameter $\beta$.

The two equilibria with label $1_{R}$ are unstable of saddle $\times$ saddle $\times$ centre type and complex saddle $\times$ centre type, with a one-parameter family of periodic

orbits around each of them. The four equilibria with index $2_{R}$ are also unstable of saddle $\times$ centre $\times$ centre type; the dimension of their associated stable and unstable manifolds is one, while the one of the centre manifold is four. Hence, there are two families of periodic orbits associated. In the $3_{R}$ case we must consider different possibilities: if $Z=\sqrt{X}$ and $\beta \in(-\infty,-0.9516) \cup(3.4525, \infty)$, or if $Z=-\sqrt{X}$ and $\beta \in(-\infty,-3.4525) \cup(0.9516, \infty)$, the four equilibrium points are totally elliptic and, therefore, there exist three families of periodic orbits associated to each of them. For some values of $\beta$ the centre component of the the $3_{R}$ points has dimension 1 , and for other beta it has full dimension (or, equivalently, in some cases there is a hyperbolic part of dimension 4 and in other cases there is no hyperbolic behaviour in the linear approximation); as a consequence these equilibria have only one family of associated periodic orbits. We want to remark that the existence of several families of periodic orbits enlarges the possibilities of candidates to nominal trajectories in spacecraft future missions.

\subsection{Zero velocity surfaces}

Fig. 16 shows the behaviour of the ZVS for values of the energy $H$ close to the ones associated to the three kinds of equilibria: $H_{1 R}=-3, H_{2 R}=2.289428$ 

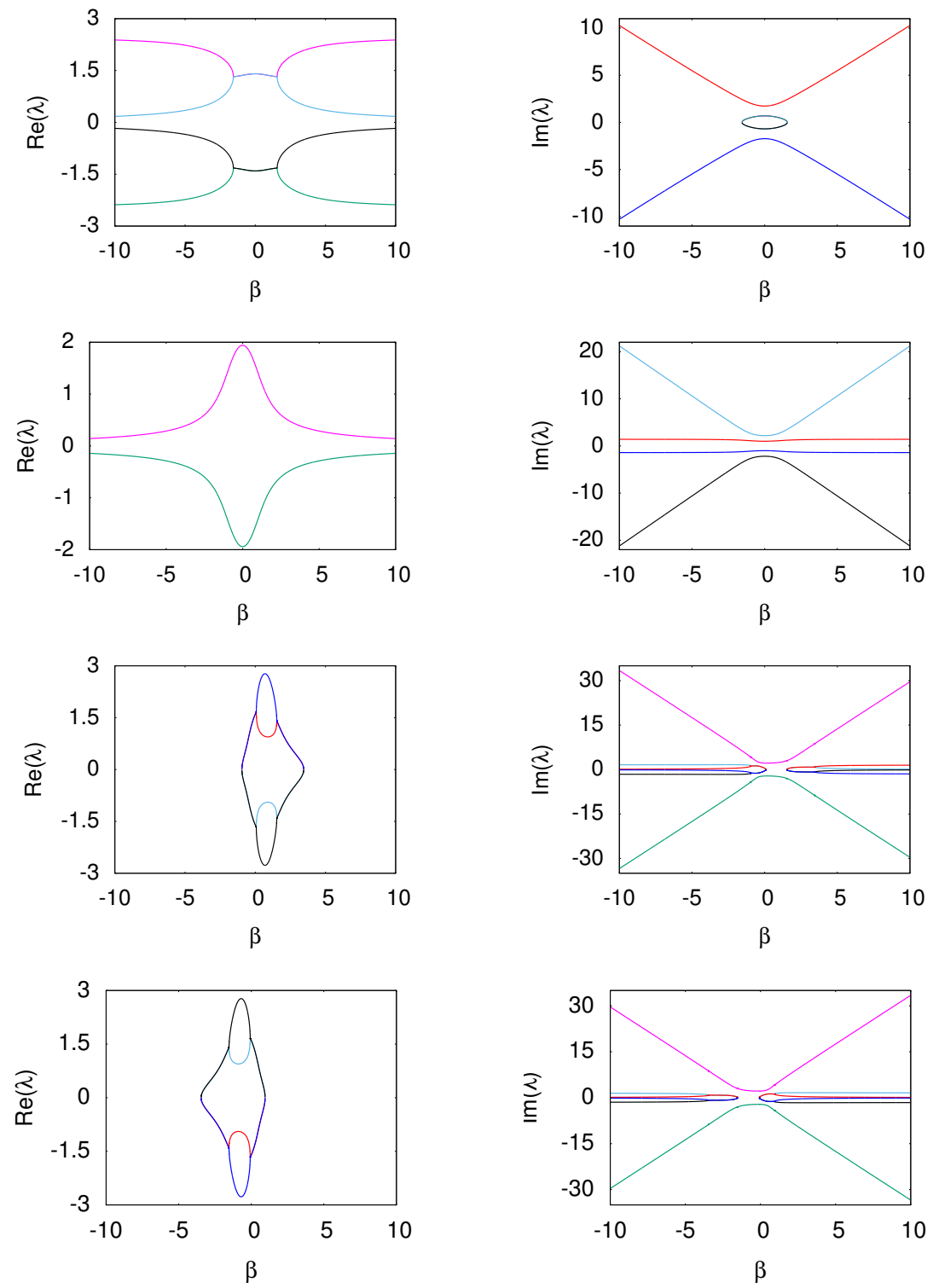

Figure 15: Real part (left column) and imaginary part (right column) of the eigenvalues associated to equilibria with labels $1_{R}$ (first row), $2_{R}$ (second row), , $3_{R}$ with $Z=\sqrt{X}$ (third row), and $3_{R}$ with $Z=-\sqrt{X}$ (fourth row) as a function of $\beta$. Only the non-zero values of the real and imaginary parts of the eigenvalues are shown.

and $H_{3 R}=1.88988$. As it follows from the figure, the maximum number of connected components of the admissible regions of motion determined by the 
ZVS is three for the first two cases and four for the last one.
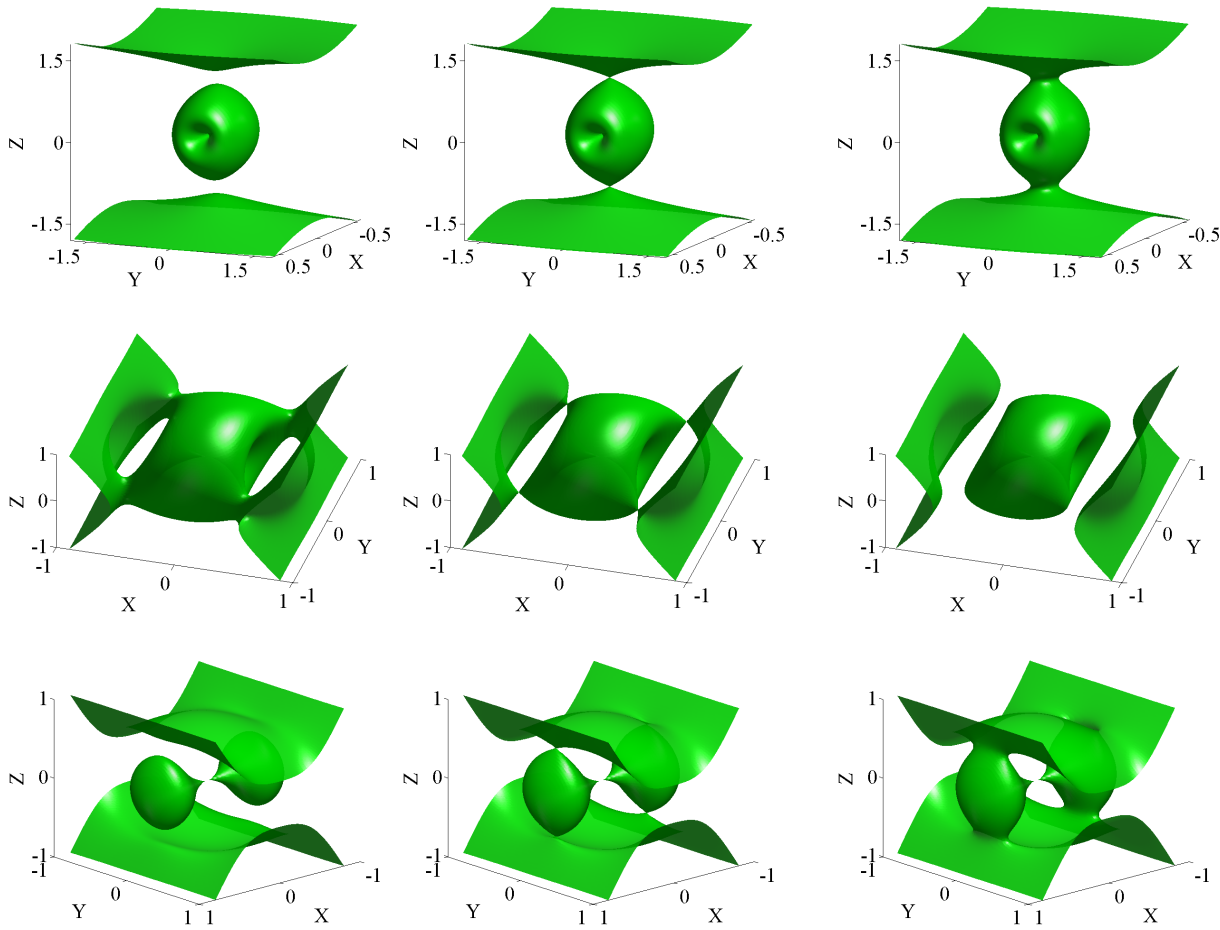

Figure 16: From top to bottom, evolution of the ZVS near the energy values associated to the equilibrium points of type $1_{R}, 2_{R}$, and $3_{R}$.

\subsection{Some families of periodic orbits}

As we have seen, in the radial case, and for all values of $\beta$, we have three kinds of equilibria: $1_{R}, 2_{R}$ and $3_{R}$. The two equilibrium points of type $1_{R}$ have a one-parameter family of periodic orbits around each of them. The second kind of equilibria is of type centre $\times$ centre $\times$ saddle, that means we have two families of periodic orbits associated to all the four $2_{R}$ points. Depending on the value of $\beta$, the $3_{R}$ equilibria can be either centre $\times$ centre $\times$ centre or centre $\times$ saddle $\times$ saddle.

For the computation of the families of periodic orbits we only consider some values of $\beta$. In each case the values are chosen such that the dimension of the 
associated centre manifold is maximal. We note that once the stability of the equilibrium point has been fixed, the qualitative behaviour of the periodic orbits emanating from the point is independent of $\beta$, although the size of the orbits changes with the value of this parameter.

\subsubsection{Periodic orbits around the $1_{R}$ equilibria}

There are two equilibria of type $1_{R}$ located at $(0,0, \pm 1)$. We will focus on the one with $Z=1$. The family of periodic orbits obtained with $\beta=2$ is displayed in Fig. 17. We stress the qualitative behaviour of the periodic orbits is independent of $\beta$. The family associated to $(0,0,-1)$ can be obtained by applying the $\mathrm{III}_{R}$ symmetry w.r.t. the origin.
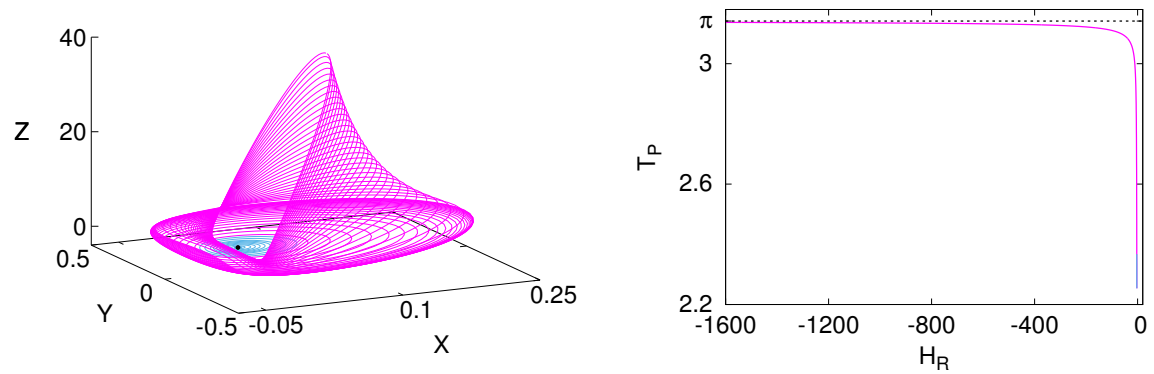

Figure 17: For $\beta=2$, family of periodic orbits emanating from the equilibrium $(0,0,1)$ of type $1_{R}(Z=1)$. The left plot shows the orbits of the family and the right one the associated characteristic curve. The colors in both figures indicate different configurations of the multipliers, which along the family goes from $B_{1}$ (blue) to $B_{4}$ (magenta).

The orbits in this family are all unstable and the type of the multipliers goes from $B_{1}$ to $B_{4}$, with the transition at $H_{R}=-3.159252$. No bifurcation has been detected at this transition. Analogously to the $1_{N}$ case with $\beta=-2$ (see Fig. 6), the orbits grow along the $Z$ direction as the energy decreases and the period tends to an upper limit value close to $\pi$.

\subsubsection{Periodic orbits around the $2_{R}$ equilibria}

There are four $2_{R}$ equilibria that located at $\left( \pm\left((2 /(9 \sqrt{3}))^{1 / 3}, \pm \sqrt{2}(2 /(9 \sqrt{3}))^{1 / 3}\right.\right.$, $0)$. We will mainly focus on the one with $X>0$ and $Y>0$. For $\beta=2$, the 
associated eigenvalues are $\lambda_{1}= \pm 4.3528 i, \lambda_{2}= \pm 1.2784 i$, and $\lambda_{3}= \pm 0.7624$, so there is one family of periodic orbits associated with $\lambda_{1}$ and another one with $\lambda_{2}$. Since the equilibrium points are in the $X-Y$ plane, we cannot determine symmetric periodic orbits (recall Table 3 for the radial case). The computation of the non-symmetric periodic orbits of these families has been done using the method explained in the Appendix taking $Z_{0}=0$. The results obtained for
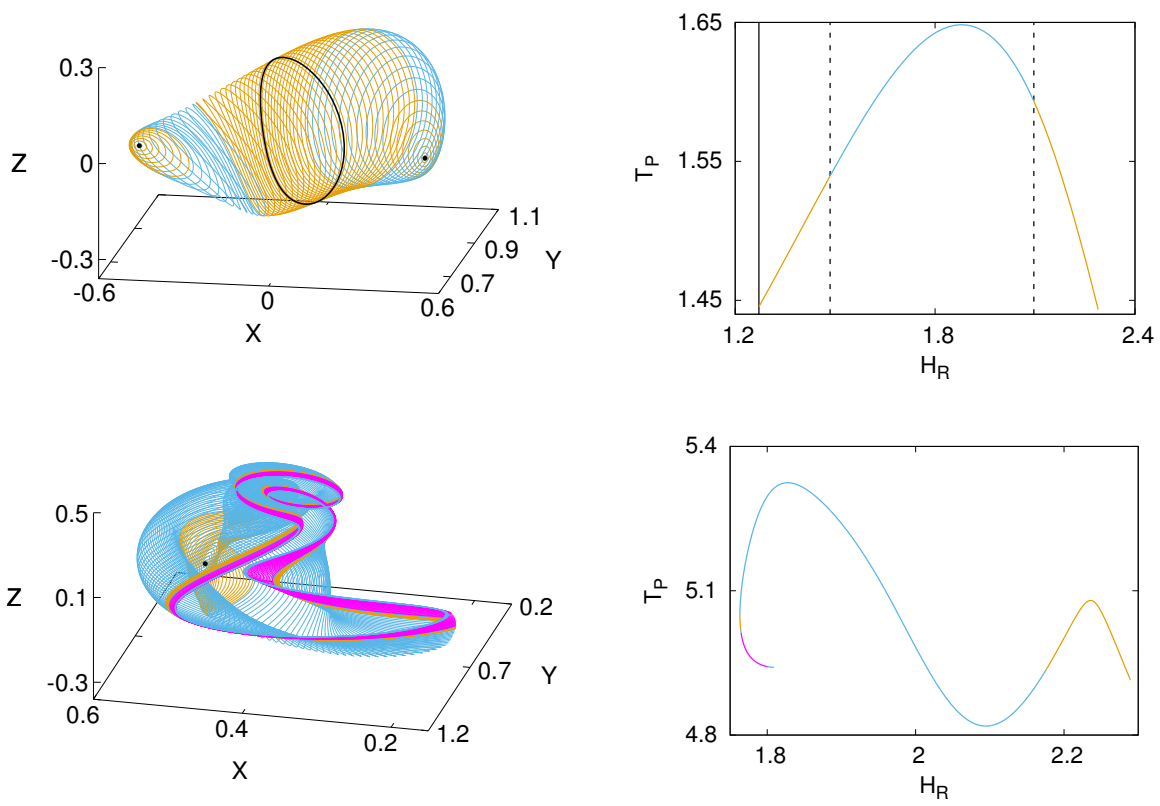

Figure 18: The two families of periodic orbits emanating from the $2_{R}$ equilibria with $Y>0$. The first row shows the results for the family associated to $\lambda_{1}$. All orbits are unstable and the transition of their type follows: $B_{2} \rightarrow B_{1} \rightarrow B_{2} \rightarrow B_{1} \rightarrow B_{2}$. Four bifurcations occur at the transition points, and indicated as vertical lines. The black line on the left, that corresponds to the orbit also displayed in black, indicates where the two branches emanating from the two $2_{R}$ equilibria meet and the type of multipliers is a degenerated center with four multipliers equal to +1 . The bottom row corresponds to the family associated to $\lambda_{2}$. All orbits are unstable and their types go through $B_{2}$ (orange) $\rightarrow B_{1}$ (blue) $\rightarrow B_{2}$ (orange) $\rightarrow B_{1}$ (blue) $\rightarrow B_{4}$ (magenta) $\rightarrow B_{1}$ (blue).

Analogous to some previous cases, such as the $1_{N}$ case with $\beta>0$ or the 
$2_{N}$ case, the family associated to $\lambda_{1}$ terminates at the equilibrium point symmetric w.r.t. the $Y$ axis. In fact, the family emanating from $\left((2 /(9 \sqrt{3}))^{1 / 3}\right.$, $\left.+\sqrt{2}(2 /(9 \sqrt{3}))^{1 / 3}, 0\right)$ and the one from $\left((2 /(9 \sqrt{3}))^{1 / 3},-\sqrt{2}(2 /(9 \sqrt{3}))^{1 / 3}, 0\right)$ are the same one, because the orbits around one point are symmetric with the ones around the other point w.r.t the $Y$ axis ( $\mathrm{II}_{R}$ symmetry). As a consequence, the characteristic curve is, in fact, the one displayed in the top row of Fig. 18 travelled twice. We note that the most left point of the curve corresponds to the orbit connecting the two families, which is a degenerated centre with the multiplier +1 with multiplicity 4 . It is the black curve on the left hand side figure that has been indicated with the vertical line in the characteristic curve.

All the orbits of this family are unstable, and for the right hand side branch, starting at $H_{R}=2.289428$ and ending at $H_{R}=1.271177$ (the connecting orbit), the transition history of the configuration type follows: $B_{2} \rightarrow B_{1} \rightarrow B_{2}$, with two periodic-doubling bifurcations at the two transitions. For the branch starting at $H_{R}=1.271177$ (that is to the left of the connecting orbit, and is symmetric of the right hand side branch w.r.t. $Y$ - axis), the transitions and the characteristic curve follow a reverse pattern as the ones of the right branch.

The orbits of the family associated with $\lambda_{2}$, together with the associated characteristic curve, are displayed in the bottom row of Fig. 18. The orbits of this family are unstable and the transitions follow the sequence: $B_{2} \rightarrow B_{1} \rightarrow$ $B_{2} \rightarrow B_{1} \rightarrow B_{4} \rightarrow B_{1}$. Two period-doubling bifurcations occur at the first and third transition, a saddle-node bifurcation occurs at the second one, and two real saddle bifurcations happen at the last two transitions, when the multipliers transit from two real saddles to a complex quadruple saddle, and vice versa.

The periodic orbits around the remaining equilibria can be obtained applying the symmetries $\mathrm{I}_{R}, \mathrm{II}_{R}$ or $\mathrm{III}_{R}$. The resulting families are displayed in Fig. 19 , together with the two connections detected between each pair of equilibria, that are symmetric w.r.t. the $Y$ axis. 


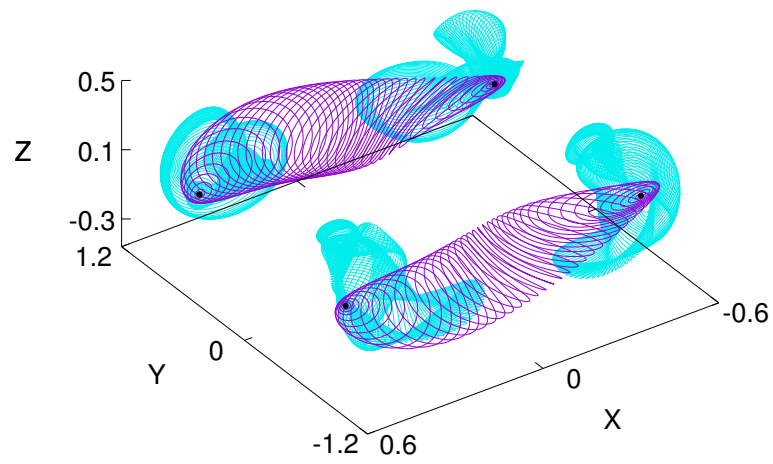

Figure 19: All the families of periodic orbits emanating from $2_{R}$ equilibria when $\beta=2$. The displayed orbits have been obtained by applying $\mathrm{I}_{R}, 2_{R}$ and $3_{R}$ symmetries from the ones computed for $X>0$ and $Y>0$.

\subsubsection{Periodic orbits around the $3_{R}$ equilibria}

There are four equilibrium points with label $3_{R}$, located at $\left( \pm(1 /(4 \sqrt{2}))^{1 / 3}\right.$, $\left.0, \pm(1 /(4 \sqrt{2}))^{1 / 6}\right)$. We recall that for the two equilibria with $Z=X$ and $\beta \in(-\infty,-0.9516) \cup(3.4525, \infty)$, and also for the two equilibria with $Z=-X$ and $\beta \in(-\infty,-3.4525) \cup(0.9516, \infty)$, the maximum dimension of the associated centre manifold is six. In both cases we have three families of periodic orbits around each equilibrium point. For the computation of the periodic orbits we have used the type $\mathrm{I}_{R}$ symmetry (which we recall is a symmetry w.r.t. the $X-Z$ plane).

First we have used $\beta=6$ and considered only the two equilibrium points with $X=Z>0$. The associated eigenvalues for this value of $\beta$ are $\lambda_{1}= \pm 29.6282 i$, $\lambda_{2}= \pm 1.4666 i$ and $\lambda_{3}= \pm 0.1381 i$. Fig. 20 shows the results obtained for the three families of periodic orbits. The first family goes through the type evolution $B_{3} \rightarrow B_{2} \rightarrow B_{3}$ with two saddle-node bifurcations and terminates in infinitesimal oscillations around the origin. The second one is stable $\left(B_{3}\right)$ and terminates at a degenerated centre, with the multiplier +1 with multiplicity 4 , while the third family goes from $B_{3}$ to $B_{2}$, after a saddle-node bifurcation at $H_{R}=1.917373$, and terminates at a degenerate saddle with the multiplier +1 
also with multiplicity 4 .

A connection between the family associated with $\lambda_{1}$ and its image w.r.t. the origin (Type $\mathrm{III}_{R}$ symmetry) has been detected. The connecting family is shown in Fig. 21.
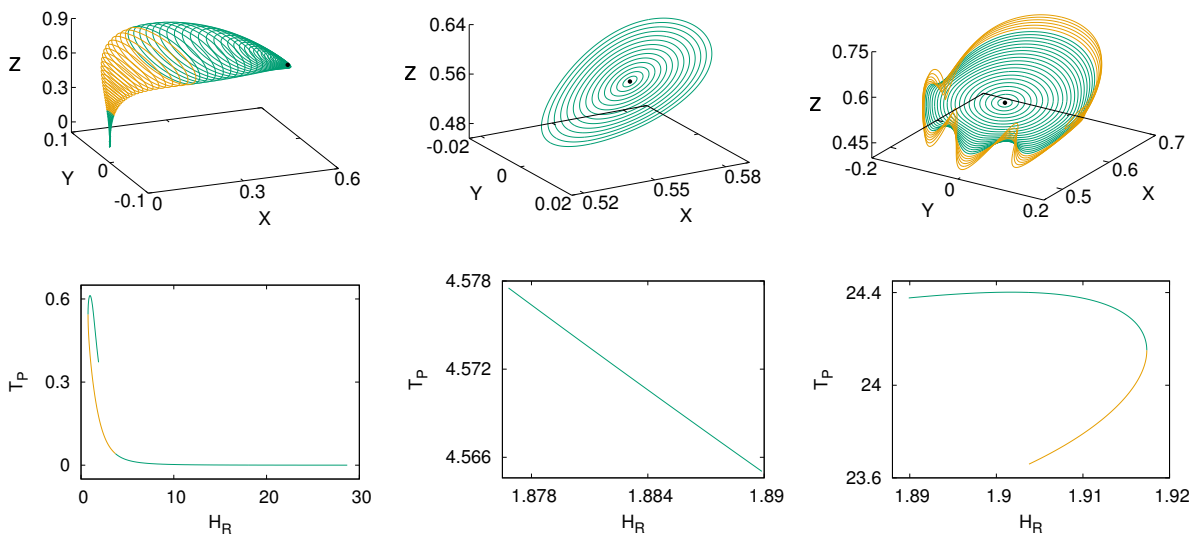

Figure 20: The three families of periodic orbits emanating from the $3_{R}$ equilibria, with $X=$ $Z>0$, associated with $\lambda_{1}$ (left), $\lambda_{2}$ (middle) and $\lambda_{3}$ (right). In the family associated with $\lambda_{1}$ the orbits are stable $\left(B_{3}\right)$ before a saddle-node bifurcation occurs at $H_{R}=0.755442$ and turn into unstable $\left(B_{2}\right)$. At $H_{R}=3.710629$ a second saddle-node bifurcation occurs and the family remains stable $\left(B_{3}\right)$ until its termination. The orbits of the family associated with $\lambda_{2}$ are stable $\left(B_{3}\right)$ and terminate at a degenerated centre at $H_{R}=1.876817$. The orbits of the family associated with $\lambda_{3}$ go from $B_{3}$ (stable) to $B_{2}$ (unstable). The transition happens at a saddle-node bifurcation at $H_{R}=1.917373$ and the family terminates at a degenerated saddle.

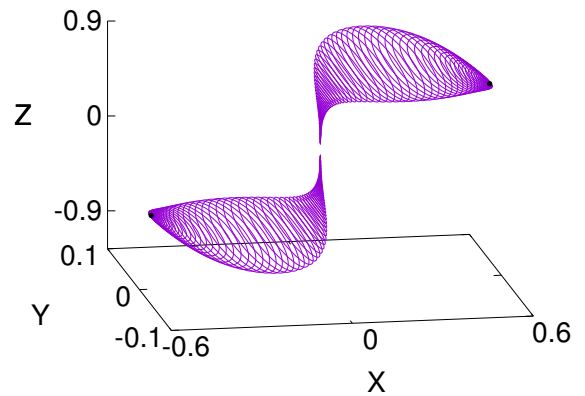

Figure 21: Connection between the family associated with $\lambda_{1}$ and its image w.r.t. the origin (Type $\mathrm{III}_{R}$ symmetry). 
Second, we have considered the two equilibrium points with $X=-Z$, for $\beta \in(-\infty,-3.4525) \cup(0.9516,+\infty)$ have also a 6 -dimensional centre manifold associated. Fig. 22 shows the three families of periodic orbits obtained in this case when $\beta=-6$. The first family (left) goes from $B_{3}$ (stable) to $B_{4}$ (unstable) type after a Neimark-Sacker bifurcation at $H_{R}=-2.046653$. Analogously to what happens to the family associated to the $1_{R}$ equilibrium point (Fig. 17), the size of the orbits becomes increases as the energy decreases, while the period tends to an upper limit close to $\pi$. The orbits of the second family (middle) are all stable and of type $B_{3}$. This family terminates at a degenerated centre when $H_{R}=1.822400$ with the multiplier +1 with multiplicity 4 . The orbits of the third family (right) are also all stable and terminate at a degenerated centre at $H_{R}=2.063967$ with the multiplier +1 with multiplicity 4 . In the second and the third families there is a monotonic dependence between the period and the energy, as is clearly shown in their associated characteristic curves.
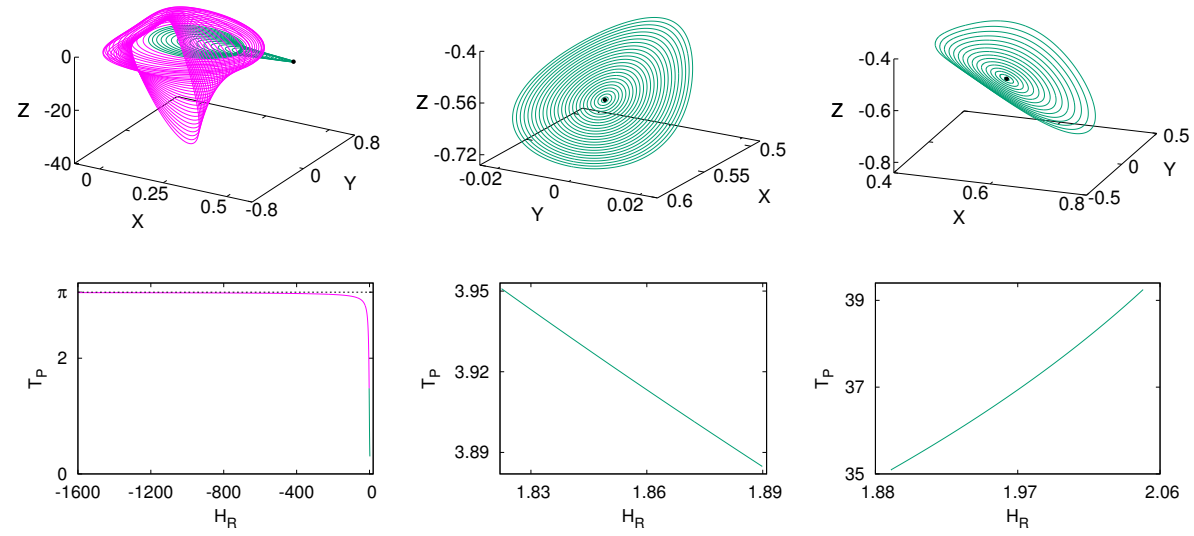

Figure 22: The three families emanating from equilibria $3_{R}$ with $Z=-X, X>0$ and $\beta=6$. The family associated with $\lambda_{1}$ (left) is stable $\left(B_{3}\right)$ up to $H_{R}=-2.046653$ where a NeimarkSacker bifurcation occurs. After this, the orbits become complex unstable $\left(B_{4}\right)$. The orbits of the other two families are stable $\left(B_{3}\right)$. The second family terminates at a degenerate centre at $H_{R}=1.822400$, while the third family ends at a degenerated real saddle at $H_{R}=2.063967$.

It might be interesting to study the case when $\beta$ only has one center component, in which case only one family of periodic orbits persists, but this has 
not been considered in the present work.

\section{The tangential case. Equilibrium points, zero velocity surfaces, periodic orbits}

\subsection{Equilibrium points}

In the tangential case the whole $Y$ - axis, except the origin, is of equilibrium points, with label $3_{T}$. These equilibrium points do not dependent on the sign $\sigma$ of $q$. There are also four additional equilibria, two on the $Z$ - axis (label $1_{T}$ ) and two on the $X$ - axis, with label $2_{T}$. Table 8 gives the results obtained. In this paper we will not consider the degenerate equilibria $3_{T}$ with $H=0$.

Table 8: Location of the equilibrium points of system 13 and value $H$ of the Hamiltonian 14 as a function of the sign $\sigma$ of the charge $q$.

\begin{tabular}{cccccc}
\hline Label & $\sigma$ & $X$ & $Y$ & $Z$ & $H$ \\
\hline $1_{T}$ & +1 & 0 & 0 & \pm 1 & -3 \\
$2_{T}$ & -1 & $\pm\left(\frac{1}{3}\right)^{1 / 3}$ & 0 & 0 & 4.326748 \\
$3_{T}$ & \pm 1 & 0 & $\neq 0$ & 0 & 0 \\
\hline
\end{tabular}

The characteristic polynomials of the two different kinds of equilibrium points considered are:

$$
\begin{array}{ll}
1_{T}: & p(\lambda)=\lambda^{6}+\beta^{2} \lambda^{4}+\left(3-3 \beta^{2}\right) \lambda^{2}-36, \\
2_{T}: & p(\lambda)=\lambda^{6}+\left(9 \beta^{2}+8\right) \lambda^{4}+\left(81 \beta^{2}-65\right) \lambda^{2}-324 .
\end{array}
$$

We have explored the evolution of the eigenvalues of the Jacobian as a function of $\beta$ which has been considered in $\beta \in[-10,10]$. Fig. 23 shows the real and imaginary parts of the six eigenvalues of the equilibrium points as a function of the parameter $\beta$.

The two equilibria with label $1_{T}$ are either of saddle $\times$ saddle $\times$ centre type or complex saddle $\times$ centre type and, consequently, there is a one-parameter family of periodic orbits around each of them. The two equilibria with index $2_{T}$ are also unstable but of saddle $\times$ centre $\times$ centre type; the dimension of their 

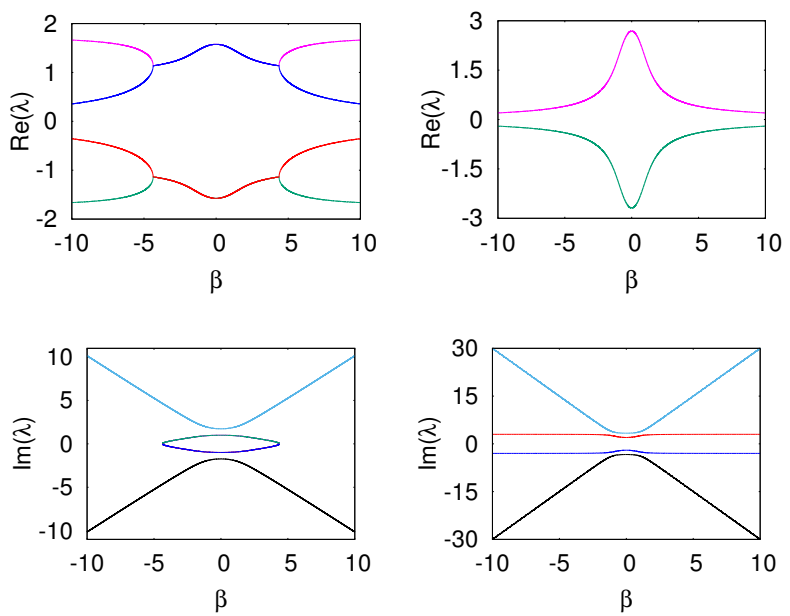

Figure 23: Real part (first row) and imaginary part (second row) of the eigenvalues associated to equilibria with labels $1_{T}$ (left) and $2_{T}$ (right) as a function of $\beta$. Only the non-zero values of the real and imaginary parts of the eigenvalues are shown.

associated stable and unstable manifolds is one, while the one of the centre manifold is four. Hence, there are two families of periodic orbits associated.
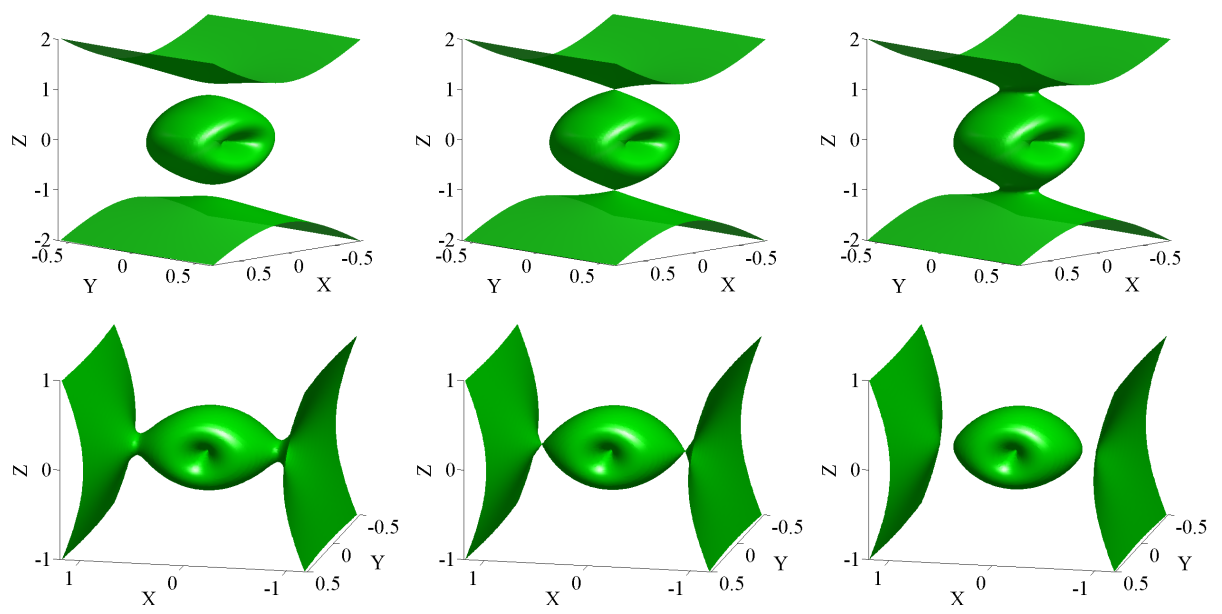

Figure 24: From top to bottom, evolution of the ZVS near the energy values associated to the equilibrium points of type $1_{T}$ and $2_{T}$. 


\subsection{Zero velocity surfaces}

In the tangential case, as we already stated, we have only considered the equilibrium points of type $1_{T}$ and $2_{T}$. According to Table 8 , there are only two equilibrium points of each type with energy values equal to $H_{1 T}=-3$ and $H_{2 T}=4.326748$. The evolutions of the ZVS for energy values close to these two ones are also displayed in Fig. 24. For the first point, the number of admissible unconnected regions is three if $H<H_{1 T}$, the three regions met at the equilibrium points when $H=H_{1 T}=-3$, and there is only one component for $H>H_{1 T}$. The evolution of the number of admissible unconnected regions, for values of the energy $H$ close to the one to the second equilibrium point, is the inverse of the preceeding one, and goes from one to three.

\subsection{Some families of periodic orbits}

We recall that in the tangential case there are two kinds of equilibrium points: two $1_{T}$ equilibria located at $(0,0, \pm 1)$, and two $2_{T}$ equilibria with coordinates $\left( \pm(1 / 3)^{1 / 3}, 0,0\right)$. For any value of $\beta$, the $1_{T}$ points have a two-dimensional centre manifold which embeds a one-parameter family of periodic orbits. The centre manifold associated to $2_{T}$ type of equilibria has dimension four which means that there are two families of periodic orbits around them. As in the preceding cases, we will focus on the equilibrium point in the first quadrant. The results for $\beta=6$, are shown in Fig. 25 .

The orbits associated with $(0,0,+1)$ are all unstable and transit from $B_{1}$ (real saddle) to $B_{4}$ (complex saddle) without any bifurcation in between. The continuation procedure is finished when the orbits become very large $(|Z|>40)$. Analogously to the families shown in Fig. 6 and Fig. 22 (top row), an upper limit close to $\pi$ for the period exists, even the size of the orbit grows rather large.

The two families associated with $\left((1 / 3)^{1 / 3}, 0,0\right)$ are also shown in Fig. 25 The orbits of the first family associated to this point are of type $B_{2}$ (unstable) and transit to $B_{3}$ (stable) after a saddle-node bifurcation at $H_{T}=3.914646$. The last orbits of the family are infinitesimal oscillations around the origin, where 

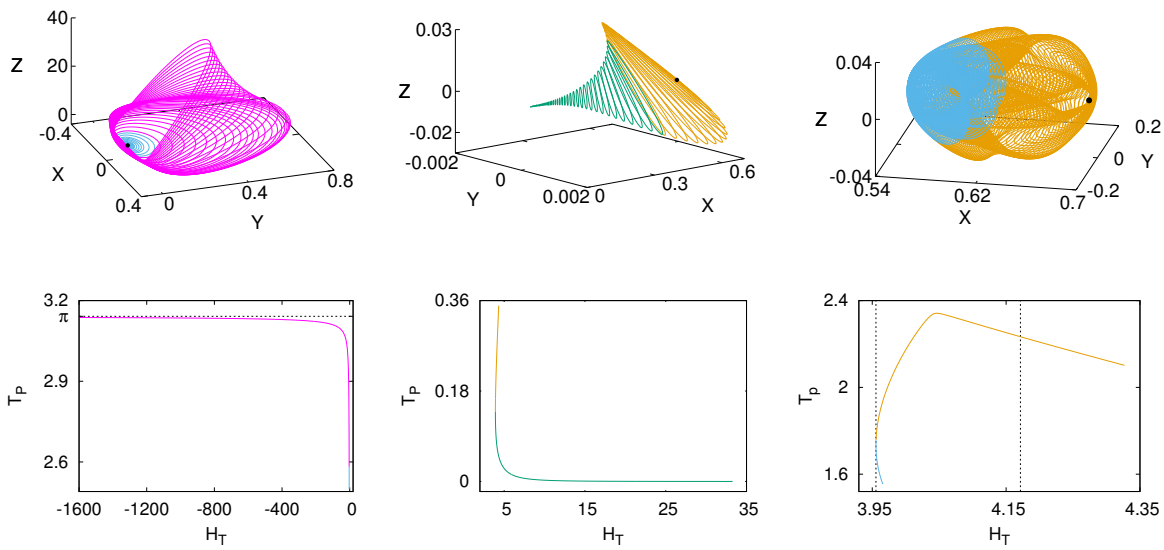

Figure 25: The three families of periodic orbits in the tangential case. The orbits of the family emanating from $(0,0,+1)$ (left) are unstable and transit from $B_{1}$ (blue) to $B_{4}$ (magenta). The two families associated with $\left((1 / 3)^{1 / 3}, 0,0\right)$ are shown in the remaining columns. The orbits of one of the families transit from $B_{2}$ (orange) to $B_{3}$ (green) type, after a saddle-node bifurcation at $H_{T}=3.914646$. The orbits of the other family transit from $B_{2}$ (orange) to $B_{1}$ (blue) when a saddle-node bifurcation occurs at $H_{T}=3.955519$. This family ends at a degenerated saddle at $H_{T}=3.965645$. At the bifurcation point when the multiplicity of the multiplier +1 becomes 4 , as indicated with the right-hand side vertical line, another family is born (see Fig. 27).

the family ends. A connection at the origin is detected between the families associated to $\left((1 / 3)^{1 / 3}, 0,0\right)$ and $\left(-(1 / 3)^{1 / 3}, 0,0\right)$, which are symmetric w.r.t. the origin (see Fig. 26).

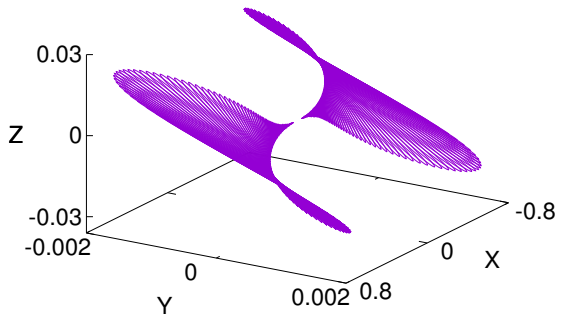

Figure 26: Connection between the families associated to $\left((1 / 3)^{1 / 3}, 0,0\right)$ and $\left(-(1 / 3)^{1 / 3}, 0,0\right)$. 
The orbits of the second family associated to $\left((1 / 3)^{1 / 3}, 0,0\right)$ are unstable and transit from $B_{2}$ to $B_{1}$ type at $H_{T}=4.326748$. The family ends as a degenerated saddle at $H_{T}=3.965645$ with the multiplier +1 with multiplicity 4 , which corresponds to the final point of the characteristic curve. One saddlenode bifurcation has been detected at $H_{T}=3.955519$. We have computed the bifurcated family born at bifurcation point at $H_{T}=4.170688$ when the multiplicity of the multiplier +1 becomes 4 (see Fig. 27).
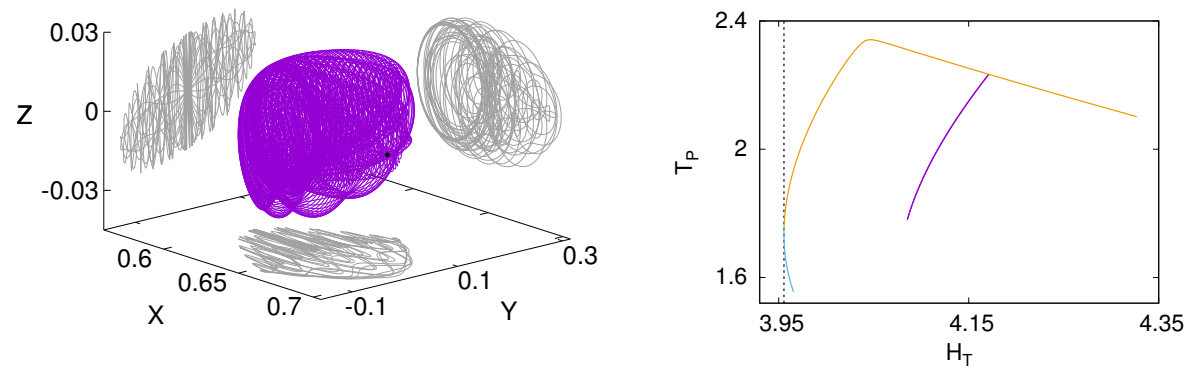

Figure 27: Bifurcated family emanating from the bifurcation point at $H_{T}=4.170688$ when four multipliers are equal to +1 appearing in the second family associated to $\left((1 / 3)^{1 / 3}, 0,0\right)$.

\section{Conclusions}

In this paper we have studied the relative dynamics of a charged spacecraft moving around a leader spacecraft provided with a rotating magnetic dipole.

The leader is assumed to move following circular orbits around a central body (for instance, the Earth). Three basic cases, for the orientation of the dipole, have been considered: normal, radial and tangential. For the equations associated to the three resulting dynamical systems, we have done an exhaustive analysis of their equilibria, and their stability behaviour with respect to the parameter $\beta$ (quotient between the mean motion of the leader and the angular velocity of the dipole). We have also explored the admissible regions of motion in the phase space, by analysing the topology of zero velocity surfaces when the energy goes through the critical values at the equilibria. 
The phase space around each equilibria has been carefully explored com-

puting the families of periodic orbits associated to them, their continuations, bifurcations, terminations, and stability properties. Four kinds of bifurcations: saddle-node bifurcation, period-doubling bifurcation, Neimark-Sacker bifurcation, real saddle bifurcation, have been detected and several bifurcated families have been computed. Furthermore, a parameterisation method, based on stroboscopic map computations, has been developed and applied to compute $2 \mathrm{D}$ invariant tori around elliptic periodic orbits. The relation between the Poincaré map method and stroboscopic map method has been established in terms of the return time. Several families of $2 \mathrm{D}$ tori have been computed, using as initial seed the linear flow around the elliptic periodic orbits.

The models considered in the paper have great potential use for future space mission applications. The overall work provides an exhaustive catalog of periodic and quasi-periodic orbits which can be interesting candidates to be used as nominal trajectories for formation flying, long time hovering missions, etc. according to their stability properties and their relative location with respect to the leader.

\section{Appendix. Computational aspects of periodic and quasi-periodic orbits}

In this section we present the methods used for the computation of the periodic and quasi-periodic orbits shown in the previously discussed.

The computation of the periodic orbits has been done using a numerical continuation method, based on a predictor method followed by a differential corrector procedure. The initial guess used to start the procedure is given by the periodic solutions of the linearised differential equations around an equilibrium point. In most cases, the symmetries of the differential equations have been used to reduce the computational cost. Some papers related with the computation and the linear analysis of periodic orbits in other dynamical models are: [5, 9 , 10, 36], and [37. 
Around the equilibrium points we can also expect Lissajous orbits filling 2D invariant tori. We can imagine them as the coupling of two periodic motions with non-resonant frequencies $\omega_{i}$ and $\omega_{j}$. Close to the equilibrium point the frequencies of the tori will be close to the ones of the periodic orbits, but the frequencies change with the amplitudes and so, they go across resonances. The proof of the existence of this Cantor set of tori follows the main idea underlying the KAM theorem (see [19]). We compute them using the parameterisation method given in Section 7.2 .

\subsection{Computation of periodic orbits around the equilibrium points}

Let $\lambda_{1}, \ldots, \lambda_{6}$ be the eigenvalues of any of the Jacobians considered. The solution of the linearised system, around the associated equilibrium point, can be written as

$$
\boldsymbol{x}(t)=c_{1} e^{\lambda_{1} t} \boldsymbol{v}_{1}+c_{2} e^{\lambda_{2} t} \boldsymbol{v}_{2}+\cdots c_{6} e^{\lambda_{6} t} \boldsymbol{v}_{6},
$$

where $\boldsymbol{v}_{i}, i=1, \ldots, 6$ are the eigenvectors and $c_{i}, i=1, \ldots, 6$ arbitrary constants.

We assume that the dimension of the centre manifold is, at least, two. Then, at least one eigenvalue $\lambda_{1}$ (together with its complex conjugate $\lambda_{4}$ ) is pure imaginary and we can write $\lambda_{1,4}= \pm \sqrt{-1} s= \pm i s$, with $s \in \mathbb{R}$. Setting $c_{2}=$ $c_{3}=c_{5}=c_{6}=0$ we get

$$
\boldsymbol{x}(t)=c_{1} e^{i s t} \boldsymbol{v}_{1}+c_{4} e^{-i s t} \overline{\boldsymbol{v}}_{1}
$$

where $\overline{\boldsymbol{v}}_{1}$ is the conjugate of $\boldsymbol{v}_{1}$. Using this linear approximation, the periodic orbit used as initial guess for the non-linear system is

$$
\boldsymbol{X}(t)=\boldsymbol{X}_{e q}+\epsilon \frac{\boldsymbol{x}(t)}{\|\boldsymbol{x}(t)\|},
$$

where $\boldsymbol{X}_{e q}$ denotes the equilibrium point, $\epsilon$ is a small parameter, that usually has been taken equal to $10^{-3}$, and $\|\boldsymbol{x}(t)\|$ is the Euclidean norm of $\boldsymbol{x}(t)$.

Once the above initial orbits have been computed, we can start the predictorcorrector scheme for the computation of the family of periodic orbits associated to $\lambda_{1}$, assuming that there are no other non-resonant imaginary eigenvalues $\left(\lambda_{j} / \lambda_{1} \notin \mathbb{Z}\right.$ for $\left.j=2,3\right)$. Of course, if the dimension of the centre manifold is 
larger than 2 , the above procedure can be done for any other pair of imaginary eigenvalues.

We consider two main kinds of symmetric periodic orbits: those that are symmetric with respect to a coordinate plane, and those which have axial symmetry. Both kinds of orbits are sketched in Fig. 28. We consider only periodic orbits such that their initial and final configurations belong to same kind of symmetry.

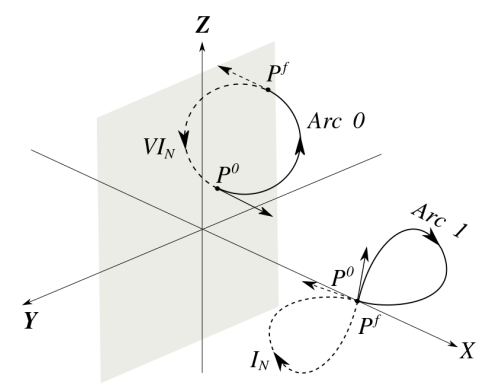

Figure 28: Sketch of the two kinds of symmetric periodic orbits: Arc 0 with coordinate plane symmetry, and Arc 1 with axial symmetry. The labels $V I_{N}$ and $I_{N}$ correspond to two of the symmetries defined in Table 2 These symmetries are used for the computation of periodic orbits integrating them during only half a period.

Let $\left(X^{0}, Y^{0}, Z^{0}, X^{\prime 0}, Y^{\prime 0}, Z^{\prime 0}\right) \equiv\left(X_{1}^{0}, X_{2}^{0}, X_{3}^{0}, X_{4}^{0}, X_{5}^{0}, X_{6}^{0}\right)$ be the initial conditions of any of these symmetric orbits. According to the kind of symmetry (as given in Tables 2,3 and 4) and symmetry element (line or plane), only three components of the initial condition are different from zero, since the initial conditions are taken on the symmetry element and the velocity at this point must be perpendicular to it.

\subsubsection{The differential corrector}

Due to the symmetries, we can assume that the initial conditions have only three non-zero components. If, for instance, the periodic orbits are symmetric ${ }_{655}$ w.r.t. the $Y-Z$ plane, the initial conditions will be $\left(0, Y^{0}, Z^{0}, X^{\prime 0}, 0,0\right)^{T}$, and we will ask that the orbit with these initial conditions intersects again the symmetry plane $X=0$ with the same non-zero components. This means that 
at after half a period (at $X=0$ ) the following final conditions must be satisfied: $Y^{\prime f}=Z^{\prime f}=0$.

Denoting by $\boldsymbol{X}=\left(X_{i}^{0}, X_{j}^{0}, X_{k}^{0}\right)^{T}$ the vector with the non-zero components of the initial condition, the non-linear system to be solved is:

$$
\left\{\begin{array}{l}
F_{1}\left(X_{i}^{0}, X_{j}^{0}, X_{k}^{0}\right)=X_{m}^{f}=0, \\
F_{2}\left(X_{i}^{0}, X_{j}^{0}, X_{k}^{0}\right)=X_{n}^{f}=0,
\end{array}\right.
$$

and $X_{k-3}^{f}=0$ or $X_{j-3}^{f}=0$. Thus, if $X_{l}^{f}=0$ is the final condition, then we have $l=k-3, m=i+3, n=j+3$ for the plane symmetry case, and $l=j(k)-3$, $\mathrm{m}=\mathrm{i}+3, n=k(j)-3$ for axial symmetry case.

The above system (21) of two equations with three unknowns can be written as

$$
F(\boldsymbol{X})=\mathbf{0} .
$$

This system is solved by means of a modified Newton's method in which, at each iteration, we minimise the Euclidean norm of the correction given by Newton's method. For $K=1,2,3, \ldots$ the equations of the iterative procedure are

$$
\begin{aligned}
\boldsymbol{X}_{K} & =\boldsymbol{X}_{K-1}+\Delta \boldsymbol{X}_{K-1}, \\
\Delta \boldsymbol{X}_{K-1} & =-G^{T}\left(G G^{T}\right)^{-1} \cdot F\left(\boldsymbol{X}_{K-1}\right),
\end{aligned}
$$

where $G$ is the Jacobian matrix of $F$ with respect to $\left(X_{i}^{0}, X_{j}^{0}, X_{k}^{0}\right)$, and is given by

$$
G=\left[\begin{array}{ccc}
\Phi_{m, i} & \Phi_{m, j} & \Phi_{m, k} \\
\Phi_{n, i} & \Phi_{n, j} & \Phi_{n, k}
\end{array}\right]-\frac{1}{X_{l}^{\prime f}} \cdot\left[\begin{array}{c}
X_{m}^{\prime f} \\
X_{n}^{\prime f}
\end{array}\right]\left[\begin{array}{lll}
\Phi_{l, i} & \Phi_{l, j} & \Phi_{l, k}
\end{array}\right],
$$

where, $\Phi$ is the $6 \times 6$ state transition matrix, solution of the linear variational 670 equations after half a period. The iterative procedure defined by 22 finishes when either $\left\|F\left(\boldsymbol{X}_{K}\right)\right\|$ or $\left\|\Delta \boldsymbol{X}_{K-1}\right\|$ is less than a certain threshold. 
For non-symmetric periodic orbits the above refinement strategy is no longer valid. In this case the periodicity condition must be adjusted to return to the same initial state after a full period. In this case we have 7 unknowns ${ }_{675} \boldsymbol{X}=\left[X^{0}, Y^{0}, Z^{0}, X^{\prime 0}, Y^{\prime 0}, Z^{\prime 0}, T\right]^{T}$ (6 state vector + period $)$, and 6 equations $\boldsymbol{X}^{f}-\boldsymbol{X}^{0}=\mathbf{0}$.

To set the initial condition we fix one of its components, for example: $Y^{0}=0$. Taking into consideration that the energy is preserved during the integration, we can eliminate one of the constraints on final state, i.e. $Z^{\prime f}-Z^{0}=0$, and as a consequence we have a system of 6 unknowns and 5 equations. To solve this non-linear system we proceed as in the symmetric case, asking at each iteration for a minimum norm correction. Now, the Jacobian $G$ in $(22)$ is the $6 \times 5$ matrix $\Phi-I d$, of which we eliminate the 6 -th row, because of the constraint $Z^{\prime f}-Z^{0}=0$, and the second column, to remove the correction on initial component $Y^{0}$.

For the computations we have used a Runge-Kutta-Fehlberg 7-8 propagator with the following parameters: maximum local truncation error, $10^{-13}$; maximum error in the determination of the final condition on the fixed plane/line, $10^{-11}$; and maximum value of $\left\|F\left(\boldsymbol{X}_{K}\right)\right\|$ or $\left\|\Delta \boldsymbol{X}_{K-1}\right\|, 10^{-10}$.

\subsubsection{The continuation method}

The computation of the families of periodic orbits has been done using a continuation method, with the arc-parameter, $s$, as continuation parameter. In this way, the curve $\left(X_{i}^{0}(s), X_{j}^{0}(s), X_{k}^{0}(s)\right)$, in the 3 -dimensional space $\left(X_{i}, X_{j}, X_{k}\right)$, is the characteristic curve of the family and satisfies the system of differential equations (see [14]),

$$
\frac{d X_{i}}{d s}=\frac{A_{1}}{A_{0}}, \quad \frac{d X_{j}}{d s}=\frac{A_{2}}{A_{0}}, \quad \frac{d X_{k}}{d s}=\frac{A_{3}}{A_{0}},
$$

where $A_{0}=\left(A_{1}^{2}+A_{2}^{2}+A_{3}^{2}\right)^{1 / 2}, A_{1}=\left(F_{X_{j}}^{1} F_{X_{k}}^{2}-F_{X_{k}}^{1} F_{X_{j}}^{2}\right), A_{2}=-\left(F_{X_{i}}^{1} F_{X_{k}}^{2}-\right.$ $\left.F_{X_{k}}^{1} F_{X_{i}}^{2}\right), A_{3}=-\left(F_{X_{i}}^{1} F_{X_{j}}^{2}-F_{X_{j}}^{1} F_{X_{i}}^{2}\right)$, and $F_{X_{i}\left(X_{j}, X_{k}\right)}^{1(2)}$ is the partial derivative of $F^{1(2)}$ with respect to $X_{i}\left(X_{j}, X_{k}\right)$.

The integration of 23 is done using an Adams-Bashforth method with one, 
two, three or four steps, depending on the number of available points on the curve. Therefore, if we suppose, for instance, that four points $\boldsymbol{X}_{r}^{0}, \boldsymbol{X}_{r+1}^{0}, \boldsymbol{X}_{r+2}^{0}$, $\boldsymbol{X}_{r+3}^{0}$ are known, the integration of 23 will provide a new point $\boldsymbol{X}_{r+4}$ near the curve. Due to the relative low accuracy of this integration procedure, the resulting point $\boldsymbol{X}_{r+4}$ must be refined by means of the differential correction method previously explained in order to get the accurate initial conditions $\boldsymbol{X}_{r+4}^{0}$ of the new periodic orbit.

With regard to the (fixed) step size $\Delta s$ used in Adams-Bashforth method for the integration of $(23)$, we have followed the automatic control strategy given in [36. From one side, $\Delta s$ must be adjusted (decreased) to make sure that the curvature of the characteristic curve is not too strong, that is, the angle between the two segments determined by three consecutive points be less than some bound (we have used 0.1rad); from the other side, if the number of iterations required by Newton's method is small (less than 3) we can speed up the computation of the characteristic curve increasing $\Delta s$. Analogously, if the number of Newton's iteration is too large (greater than 8) it is convenient to decrease $\Delta s$. The above step-size control must be implemented accurately, adding or removing points of the characteristic curve, since Adams-Bashforth method is a fixed step-size propagator. For other developments and implementations of the arc-length continuation method see [11, 12, 13].

We stop the continuation procedure when either:

- The required step size $\Delta s$ is smaller than a certain amount $\left(10^{-6}\right)$, which usually happens when the family is close to a bifurcation or to its natural termination (for instance, at another equilibrium point).

- The size of the orbits becomes very large (modulus of the position vector larger than 50 units). This is because we are mainly interested in the application of the orbits obtained to formation flying, in which the follower is usually required to be in the proximity of the leader.

We remark that the arc-length continuation procedure is able to jump some bifurcations, remaining along the original family, while in other cases it follows 

of the stability parameters of the orbits.

\subsection{Computation of $2 D$ invariant tori}

In this section we explain the method used for the computation of $2 \mathrm{D}$ invariant tori around periodic orbits of elliptic type. This kind of orbits appear, for instance, around the periodic orbits associated to the equilibria with label $3_{N}$ (see Fig. 11).

There are two commonly used numerical methods for the computation of 2D invariant tori [3]: the stroboscopic map method [10], that we use in our implementation, and the Poincaré map method [21, which, according to [16], are also called large matrix methods. Let us show the connection between them.

Consider, for instance, the differential equations (8) of the normal case, and write these equations as the first order system,

$$
\dot{\chi}=f(\chi), \quad \chi=\left(X, Y, Z, X^{\prime}, Y^{\prime}, Z^{\prime}\right) \in \mathbb{R}^{6} .
$$

According to [10, the computation of a $2 \mathrm{D}$ torus can be done looking for a parameterisation of it, $\psi\left(\theta_{1}, \theta_{2}\right)$, with

$$
\begin{aligned}
& \psi: \quad \mathbb{R}^{2} \quad \longrightarrow \quad \mathbb{R}^{6} \\
& (\xi, \eta) \longrightarrow \psi(\xi, \eta),
\end{aligned}
$$

where $\psi$ is $2 \pi$-periodic in both arguments $\xi, \eta$, and invariant under the flow associated to 24. This is:

$$
\psi\left(\xi+t \omega_{1}, \eta+t \omega_{2}\right)=\phi_{t}(\psi(\xi, \eta)), \quad \forall t \in \mathbb{R}, \quad \forall \xi, \eta \in[0,2 \pi],
$$

where $\omega_{1}, \omega_{2}$ are the frequencies of the torus, and $\phi_{t}$ denotes the flow associated to the differential system. So $\phi_{t}(\psi(\xi, \eta))$ is the image under the flow of the point $\psi(\xi, \eta)$ after $t$ time units.

In order to reduce the dimension of the problem, one of the parameters can be fixed, for instance taking $\eta=\eta_{0}=0$. This is equivalent to fix a curve on the torus, $\varphi(\xi)=\psi(\xi, 0)$, that must be invariant under $\phi_{2 \pi / \omega_{2}}$. So we have,

$$
\varphi(\xi+\rho)=\phi_{T}(\varphi(\xi))
$$


where $\rho=2 \pi \omega_{1} / \omega_{2}$ is the rotation number in radians of the invariant curve $\varphi$, and $T=2 \pi / \omega_{2}$. Once the parameterisation of the curve $\varphi$ has been computed, the torus $\psi$ can be determined by means of (see [10])

$$
\psi(\xi, \eta)=\phi_{\frac{\eta-\eta_{0}}{2 \pi} T}\left(\varphi\left(\xi-\frac{\eta-\eta_{0}}{2 \pi} \rho\right)\right) .
$$

Let us assume that the invariant curve $\varphi: \mathbb{S}^{1} \rightarrow \mathbb{R}^{6}$ that we want to compute has rotation number $\rho$, and its truncated Fourier expansion is,

$$
\varphi(\xi)=\boldsymbol{C}_{0}+\sum_{k=1}^{M}\left(\boldsymbol{C}_{k} \cos (k \xi)+\boldsymbol{S}_{k} \sin (k \xi)\right),
$$

where $\boldsymbol{C}_{0} \in \mathbb{R}^{6}, \boldsymbol{C}_{k} \in \mathbb{R}^{6}$, and $\boldsymbol{S}_{k} \in \mathbb{R}^{6}$ are Fourier coefficients to be determined.

The invariance equation for $\varphi$ can be written as,

$$
\varphi(\xi+\rho)=\phi_{T(\xi)}(\varphi(\xi))
$$

where the return-time $T(\xi)$ is fixed to be the period (or the normal period) of the periodic orbit for the stroboscopic map method, while for the Poincaré map method depends on $\xi$ and it is different at each point.

\subsubsection{Indeterminations in the parameterisation of $\phi$}

There are two indeterminations in the Fourier representation of $\varphi(\xi)$. One is due to the location of the invariant curve on the torus, since for any value of $\eta_{0} \in[0,2 \pi]$, not only for $\eta_{0}=0$, the curve $\varphi(\xi)=\psi\left(\xi, \eta_{0}\right)$ satisfies the invariance equation (26). This indetermination can be avoided by fixing one coordinate of $\boldsymbol{C}_{0}$. We note that this coordinate needs to be chosen taking into consideration the geometry of the torus.

The other indetermination is due to possible phase shifts $\xi_{0}$ in the Fourier representation. This is, if $\varphi(\xi)$ satisfies the invariant equation (29), then $\varphi(\xi+$ $\left.\xi_{0}\right), \forall \xi_{0} \in \mathbb{R}$ also does. In fact we have,

$$
\varphi\left(\xi+\xi_{0}\right)=\boldsymbol{C}_{0}+\sum_{k=1}^{N_{f}}\left(\tilde{\boldsymbol{C}}_{k} \cos (k \xi)+\tilde{\boldsymbol{S}}_{k} \sin (k \xi)\right),
$$

750

with another representation, $\tilde{\boldsymbol{C}}_{k}=\boldsymbol{C}_{k} \cos \left(k \xi_{0}\right)+\boldsymbol{S}_{k} \sin \left(k \xi_{0}\right)$ and $\tilde{\boldsymbol{S}}_{k}=\boldsymbol{S}_{k} \cos \left(k \xi_{0}\right)-$ $\boldsymbol{C}_{k} \sin \left(k \xi_{0}\right)$. 
The phase shift indetermination can be avoided setting one component of $\boldsymbol{C}_{1}$ or $\boldsymbol{S}_{1}$ equal to zero by an appropriate selection of the value of $\xi_{0}$ (see [10]), and with the Fourier coefficients updated to $\tilde{\boldsymbol{C}}_{k}, \tilde{\boldsymbol{S}}_{k}, k=1 \div N_{f}$, by means of (30). The index $J$ of the component is chosen such that,

$$
\left\|\boldsymbol{C}_{1}^{J}, \boldsymbol{S}_{1}^{J}\right\|_{2}=\max _{j=1,2}\left\|\boldsymbol{C}_{1}^{j}, \boldsymbol{S}_{1}^{j}\right\|_{2} .
$$

Between $\boldsymbol{C}_{1}^{J}$ and $\boldsymbol{S}_{1}^{J}$ we choose the one with maximum absolute value. Once both selections have been done, then one unknown $\boldsymbol{C}_{1}^{J}$ (or $\boldsymbol{S}_{1}^{J}$ ) and the corresponding column can be removed from the Jacobian.

\subsubsection{The system of equations}

When it exists, the saddle component associated to a torus can introduce instability in the numerical integration. To overcome this problem a multiple shooting procedure is used (see [10]). In this way, instead of computing just $\varphi(\xi)$, several curves, $\varphi_{j}(\xi), j=0 \div m-1$, are computed. They are defined by,

$$
\begin{aligned}
\varphi_{j+1}(\xi) & =\phi_{T / m} \varphi_{j}(\xi), \quad j=0 \div m-2, \\
\varphi_{0}(\xi+\rho) & =\phi_{T / m} \varphi_{m-1}(\xi)
\end{aligned}
$$

where $\varphi_{0}$ is the original curve, and the value of $m$ depends on the instability of the torus. We have used values of $m$ between 2 and 4 . All the curves are evaluated in a discrete set of points,

$$
\xi_{i}=i \frac{2 \pi}{1+2 N_{f}}, \quad i=0, \ldots, 2 N_{f}
$$

Since we always fix the value of the energy $H$ of the torus, one more equation is added to the above system. In this way, the final system to be solved using the modified Newton's method is,

$$
\begin{aligned}
H\left(\varphi_{0}(0)\right)-H & =0, \\
\varphi_{j+1}\left(\xi_{i}\right)-\phi_{T / m} \varphi_{j}\left(\xi_{i}\right) & =0, \quad j=0 \div m-2, \\
\varphi_{0}\left(\xi_{i}+\rho\right)-\phi_{T / m} \varphi_{m-1}\left(\xi_{i}\right) & =0
\end{aligned}
$$

where, in principle, the unknowns are $\rho, T, H$ and the Fourier coefficients $\boldsymbol{A}$ of dimension $6 m \times\left(2 N_{f}+1\right)$. The coefficients $\boldsymbol{A}$ can be expressed as $\boldsymbol{A}=$ 
$\left(\boldsymbol{A}^{0}, \ldots, \boldsymbol{A}^{j}, \ldots, \boldsymbol{A}^{m-1}\right)$ with $\boldsymbol{A}^{j}=\left(\boldsymbol{C}_{0}^{j}, \boldsymbol{C}_{1}^{j}, \ldots, \boldsymbol{C}_{N_{f}}^{j}, \boldsymbol{S}_{1}^{j}, \ldots, \boldsymbol{S}_{N_{f}}^{j}\right)$ for $j=$ $0 \div m-1$.

Since we look for torus embedded in two-parameter families, specified by at least two parameters among $(\rho, T, H)$, we always remove one unknown, usually $\rho$ or $H$.

\subsubsection{Initial guess for the curve $\varphi$}

The computation of an initial guess for the curve $\varphi$ is done following the ideas given in 10. We consider a periodic orbit with centre part, this is, with at least one pair of the associated eigenvalues on the unit circle, and use the linear flow around the elliptic periodic orbit to compute the initial seed for the invariant curve.

Let $M$ be the monodromy matrix associated to an initial point $\chi_{0}$ of the periodic orbit with period $T_{p}$, that is, $M=D \phi_{T_{p}}\left(\chi_{0}\right)$. The linear flow around $\chi_{0}$ is given by,

$$
L_{\phi_{T_{p}}}^{\chi_{0}}(\chi)=\chi_{0}+M\left(\chi-\chi_{0}\right)
$$

Let $\cos \gamma+i \sin \gamma$ be an unitary eigenvalue of $M$ and $\boldsymbol{v}=\boldsymbol{v}_{r}+i \boldsymbol{v}_{i}$ the associated eigenvector. Then we have,

$M\left(\boldsymbol{v}_{r}+i \boldsymbol{v}_{i}\right)=(\cos \gamma+i \sin \gamma)\left(\boldsymbol{v}_{r}+i \boldsymbol{v}_{i}\right)=\boldsymbol{v}_{r} \cos \gamma-\boldsymbol{v}_{i} \sin \gamma+i\left(\boldsymbol{v}_{r} \sin \gamma+\boldsymbol{v}_{i} \cos \gamma\right)$

that can be rewritten as,

$$
M\left[\boldsymbol{v}_{r}, \boldsymbol{v}_{i}\right]=\left[\boldsymbol{v}_{r}, \boldsymbol{v}_{i}\right] R(\gamma), \quad \text { with } \quad R(\gamma)=\left(\begin{array}{cc}
\cos \gamma & \sin \gamma \\
-\sin \gamma & \cos \gamma
\end{array}\right)
$$

where $\left[\boldsymbol{v}_{r}, \boldsymbol{v}_{i}\right]$ denotes the $6 \times 2$ matrix with columns $\boldsymbol{v}_{r}, \boldsymbol{v}_{i}$.

Let us define a curve close to the periodic orbit by means of,

$$
\varphi(\xi)=\chi_{0}+\epsilon\left(\boldsymbol{v}_{r} \cos \xi-\boldsymbol{v}_{i} \sin \xi\right)
$$

where $\epsilon$ is the distance from $\varphi$ to the periodic orbit, and $\xi \in[0,2 \pi]$. 
The curve $\varphi$ is invariant under $L_{\phi_{T_{p}}}^{\chi_{0}}$, since

$$
\begin{aligned}
L_{\phi_{T_{p}}}^{\chi_{0}}(\varphi(\xi)) & =\chi_{0}+M\left[\boldsymbol{v}_{r}, \boldsymbol{v}_{i}\right]\left(\begin{array}{cc}
\cos \xi & \sin \xi \\
-\sin \xi & \cos \xi
\end{array}\right)\left(\begin{array}{l}
\epsilon \\
0
\end{array}\right) \\
& =\chi_{0}+\left[\boldsymbol{v}_{r}, \boldsymbol{v}_{i}\right] R(\gamma) R(\xi)\left(\begin{array}{l}
\epsilon \\
0
\end{array}\right)=\chi_{0}+\left[\boldsymbol{v}_{r}, \boldsymbol{v}_{i}\right] R(\gamma+\xi)\left(\begin{array}{l}
\epsilon \\
0
\end{array}\right) \\
& =\varphi(\gamma+\xi),
\end{aligned}
$$

where $\gamma$ is the rotation number in radians, and the two basic frequencies of the associated torus can be approximated by $\left(\gamma / T_{p}, 2 \pi / T_{p}\right)$.

We remark that the above equation 36 is the linearised version of the invariance equation 26 with $T$ taken as the period of the periodic orbit. Since it defines an invariant curve, with $T$ and $\rho$ close to those of the periodic orbit, the initial guess of $\varphi$ can be given by,

$$
\begin{aligned}
H & =H\left(\chi_{0}\right), \quad T=T_{p}, \quad \rho=\gamma \\
\boldsymbol{C}_{0} & =\chi_{0}, \quad \boldsymbol{C}_{1}=\epsilon \boldsymbol{v}_{r}, \quad \boldsymbol{S}_{1}=-\epsilon \boldsymbol{v}_{i}, \quad \boldsymbol{C}_{k}=\boldsymbol{S}_{k}=0 \quad \text { for } \quad k>2 .
\end{aligned}
$$

When the multiple shooting is applied, the Fourier coefficients with $k=0,1$ of the intermediate curves $\varphi_{j}$ are given by,

$$
\varphi_{j}(\xi)=\phi_{j \frac{T_{p}}{m}} \chi_{0}+\epsilon\left(\boldsymbol{v}_{r}^{j} \cos \xi-\boldsymbol{v}_{i}^{j} \sin \xi\right)
$$

where $\boldsymbol{v}_{(r, i)}^{j}=D \phi_{j T_{p} / m} \boldsymbol{v}_{(r, i)}^{j}$.

There is another option for the initial guess of the curve $\tilde{\varphi}(\eta)$. It is the one associated to the centre part of the periodic orbit with $T$ taken as the normal period of the centre part. Since this option has not been used in our computations we omit the details on how to implement it. Further information can be found in $[10]$.

\subsubsection{The differential corrector}

System 32 is solved iteratively using the equations given in (22). The iterations stop when either $\|F\|<10^{-10}$ or $\left\|\Delta \boldsymbol{X}_{k}\right\|<10^{-10}$ is satisfied. 
The differential of the first energy equation w.r.t. to the unknowns (in fact, only $\boldsymbol{C}_{0}^{0}$ ) can be obtained easily by chain rule. The Jacobian $G$ of the remaining equations is given by,

$$
G=\left[\begin{array}{lll}
G_{A} & G_{\rho}\left(G_{H}\right) & G_{T}
\end{array}\right],
$$

where the last two columns are the differential w.r.t. two unknowns among $(\rho, T, H)$, and $G_{A}$ is the differential associated to the Fourier coefficients $\boldsymbol{A}$. This is,

$$
G_{A}=\left[\begin{array}{c}
G_{A}^{1} \\
G_{A}^{2} \\
\vdots \\
G_{A}^{6\left(2 N_{f}+1\right)}
\end{array}\right],
$$

where the sub-matrices $G_{A}^{i}, i=0, \ldots, 2 N_{f}$ evaluated at point $\xi_{i}$ are given by,

$$
G_{A}^{i}=\left.\frac{D \boldsymbol{F}}{D \boldsymbol{A}}\right|_{\xi_{i}}=\left[\begin{array}{c}
\frac{D \varphi_{j+1}\left(\xi_{i}\right)}{D \boldsymbol{A}}-\Phi \frac{D \varphi_{j}\left(\xi_{i}\right)}{D \boldsymbol{A}} \\
\frac{D \varphi_{0}\left(\xi_{i}+\rho\right)}{D \boldsymbol{A}}-\Phi \frac{D \varphi_{m-1}\left(\xi_{i}\right)}{D \boldsymbol{A}}
\end{array}\right], \quad j=0 \div m-2,
$$

being $\Phi$ the $6 \times 6$ state transition matrix evaluated after a time-interval $T / m$. When $m=1$ (no multiple shooting is applied) only the second row remains. Both $\frac{D \varphi_{0}\left(\xi_{i}+\rho\right)}{D \boldsymbol{A}}$ and $\frac{D \varphi_{j}\left(\xi_{i}\right)}{D \boldsymbol{A}}$ are sparse matrices, where the components associated to the corresponding Fourier coefficients $\boldsymbol{A}^{j}$ (the remaining ones are zeros) can be expressed with only $2 N_{f}+1$ diagonal entries $B_{k}$ :

$$
\left[\begin{array}{cccc}
B_{0} & & & \\
& B_{1} & & \\
& & \ddots & \\
& & & B_{2 N_{f}}
\end{array}\right] \text {. }
$$

The matrices $B_{i}$, of dimension $6 \times\left(2 N_{f}+1\right)$, can be expressed as,

$$
B_{i}=\left[\begin{array}{llll}
B_{i 1} & & & \\
& B_{i 2} & & \\
& & . . & \\
& & & B_{i 6}
\end{array}\right]
$$


where $B_{i l}=\left[1 \cos (\alpha) \ldots \cos \left(N_{f} \alpha\right) \sin (\alpha) \ldots \sin \left(N_{f} \alpha\right)\right]$ for $l=1 \div 6 ; \alpha=\xi_{i}+\rho$ and $\alpha=\xi_{i}$ for $\frac{D \varphi\left(\xi_{i}+\rho\right)}{D \boldsymbol{A}}$ and $\frac{D \varphi\left(\xi_{i}\right)}{D \boldsymbol{A}}$, respectively.

We note that during the iterative procedure the number of frequencies $N_{f}$ of the truncated Fourier expansion can change according to the accuracy to be achieved. The value is chosen in such a way that the maximum norm of the last $N_{f} / 4$ coefficients be one order of magnitude smaller than the required tolerance. If this condition is not fulfilled, then we double it to $2 N_{f}$ and initially setting $\boldsymbol{C}_{k}=0$ and $\boldsymbol{S}_{k}=0$ for $k=N_{f}+1, \ldots, 2 N_{f}$. We have usually started with $N_{f}=16$, which is enough for a curve with a regular shape such as a circle or ellipse. This value needs to be increased for curves with more complex shape. However, to avoid too large systems, we have set the maximum of $N_{f}$ to be 128 .

As an important remark about the implementation of the described procedure we want to mention that the computation of the $\cos (k \alpha)$ and $\sin (k \alpha)$ values, for $k=1, . ., 2 N_{f}$, has been done using the stable trigonometric recurrences given in 39. This avoids low accuracies when the arguments are large, moreover it saves CPU time.

Another remark is that we could "fall back" to the starting periodic orbit, since the periodic orbit itself is also a solution of the system with $\boldsymbol{C}_{0}=0, \boldsymbol{C}_{K}=$ $0, \boldsymbol{S}_{k}=0$. To overcome this problem we have fixed a non-zero component of $\boldsymbol{C}_{0}$. This, in principle, only needs to be done for the computation of the first torus, since during the continuation, the rotation number $\rho$ and the return time $T$ are varied, and therefore they are different from the ones of the periodic orbit.

Finally, as it has already been previously stated, two more unknowns need to be removed to avoid the curve indetermination and the phase shift indetermination. Again, this it can be done by eliminating the corresponding columns from $G$.

\subsubsection{Continuation of the $2 D$ tori family}

We use a similar predictor-corrector scheme for the continuation of the tori family as the one described in section 7.1 .2 for the continuation of periodic orbits. Once an invariant 2D torus is computed close to an elliptic periodic 
orbit, the continuation is done along its tangential space, that is the kernel of the system $\operatorname{Ker}(D F)$. Thus, let us assume a torus $\boldsymbol{X}^{k}$ is computed. The prediction step of a new torus is given by $\boldsymbol{X}^{k+1}=\boldsymbol{X}^{k}+\Delta s \cdot \boldsymbol{v}$, with $\boldsymbol{v} \subset \operatorname{Ker}(D F),\|\boldsymbol{v}\|=1$, based Linear Least Squares solver (LAPACK routine DGELSY in [1]) is used 
to solve the system defined by Equation 32 with least-norm corrections.

The value of the continuation step $\Delta s$ is adjusted within a certain interval ${ }_{845}$ (for instance $\left[10^{-5}, 10^{-2}\right]$ ). If the number of iterates of Newton's method is small (less than three), we multiply it by two, and if the refinement fails to converge or the number of iteration is large (greater than eight), we divide it by two and we start again the procedure using as prediction the tangential space of the last successful computed torus. Moreover, to guarantee a smooth continuation, we ask the angle between the last three curves be less than a given tolerance (we use $15^{\circ}$ ). If it exceeds this value, we divide $\Delta s$ by 2 and restart the prediction from the previous torus. For the computation of the angle, we consider two unknowns among $(\rho, T, H)$ and the remaining constant term $\boldsymbol{C}_{0}$ despite of $\boldsymbol{C}_{0}^{I}$.

We repeat this process until one of the following conditions is satisfied:

1. The continuation step is smaller than the lower bound allowed $\left(10^{-6}\right)$.

2. The magnitude of Fourier coefficients with $k \geq 1$ is smaller than the accuracy required by the refinement $\left(10^{-10}\right)$. This means we end up at a periodic orbit.

3. The number, $N_{f}$, of Fourier modes required is larger than the maximum value allowed (128). This usually happens when we are close to the resonance or the shape of the curve becomes very complex and many times we are able to jump the resonance.

A trick to jump through possible resonances during the continuation is by tuning the step $\Delta s$ and the value of $N_{f}$ which can be applied when the procedure is convergent but $N_{f}$ needs to be increased. During three iterations we simply allow to start again with $N_{f}$ unchanged and the step size increased to $1.3 \Delta s$. If the iterations fail to converge, we decrease $\Delta s=\Delta s / 2$ and restart with $N_{f}=2 N_{f}$. Note that the value of $N_{f}$ might change during the continuation, so the dimension of the Fourier coefficients $\left(\boldsymbol{C}_{k}, \boldsymbol{S}_{k}\right)$ needs to be specified carefully for the new curve. 
7.2.6. The relation between the two parameterisation methods for the computation of $2 D$ invariant tori

In this section we study the relation between the stroboscopic map method and the Poincaré map method for the computation of $2 \mathrm{D}$ invariant tori. The study is done looking at the return times required by both methods and assuming that no multiple shooting is used.

Let $\varphi$ be an invariant curve computed using the stroboscopic map method and $\bar{\varphi}$ another one with the same rotation number associated to an elliptic periodic orbit. We assume that they are close, so for each point $\varphi(\xi)$ on the curve $\varphi$ there is a point $\bar{\varphi}(\xi)$ close to $\varphi(\xi)$ and on the curve $\bar{\varphi}$ such that,

$$
\varphi(\xi)=\phi_{\tau(\xi)}(\bar{\varphi}(\xi))
$$

where $\tau(\xi)$ is a small time deviation (positive or negative) depending on $\xi$.

Looking at the left-hand side of the invariance equation (26), and using equation 40, we obtain,

$$
\varphi(\xi+\rho)=\phi_{\tau(\xi+\rho)}(\bar{\varphi}(\xi+\rho))=\phi_{\tau(\xi+\rho)} \circ \phi_{\bar{T}(\xi)}(\bar{\varphi}(\xi))=\phi_{\tau(\xi+\rho)+\bar{T}(\xi)}(\bar{\varphi}(\xi)) .
$$

Similarly, substituting equation 40 in the right-hand side of equation 26 we get,

$$
\phi_{T}(\varphi(\xi))=\phi_{T} \circ \phi_{\tau(\xi)}(\bar{\varphi}(\xi))=\phi_{T+\tau(\xi)}(\bar{\varphi}(\xi)) .
$$

Hence, the relation between $T$ and $\bar{T}(\xi)$ is $\tau(\xi+\rho)+\bar{T}(\xi)=T+\tau(\xi)$, which can be rewritten as the homological equation,

$$
\tau(\xi)-\tau(\xi+\rho)=\bar{T}(\xi)-T,
$$

and since both sides of the above equation have zero-average, we have

$$
T=\frac{1}{2 \pi} \int_{0}^{2 \pi} \bar{T}(\xi) d \xi
$$

The homological equation can be solved using Fourier series

$$
\begin{aligned}
\bar{T}(\xi) & =\sum_{k \in \mathbb{Z}} \bar{T}_{k} e^{2 \pi i k \xi}, & \tau(\xi) & =\sum_{k \in \mathbb{Z}} \tau_{k} e^{2 \pi i k \xi}, \\
\bar{T}_{0} & =T, & \tau_{0} & =0 .
\end{aligned}
$$


where the $\bar{T}_{0}$ and $\tau_{0}$ are the constant terms of the series. The coefficients $\bar{T}_{k}$ can be computed by evaluating $\bar{T}(\xi)$ in a regular grid and using a FFT. Moreover we have,

$\tau(\xi)-\tau(\xi+\rho)=\sum_{k \in \mathbb{Z}}\left(\tau_{k} e^{2 \pi i k \xi}-\tau_{k} e^{2 \pi i k(\xi+\rho)}\right)=\sum_{k \in \mathbb{Z}} \tau_{k}\left(1-e^{2 \pi i k \rho}\right) e^{2 \pi i k \xi}=\sum_{k \in \mathbb{Z}} \bar{T}_{k} e^{2 \pi i k \xi}$.

Hence for $k \neq 0$ we have,

$$
\tau_{k}=\frac{\bar{T}_{k}}{1-e^{2 \pi i k \rho}} .
$$

Since $\tau_{0}$ is a free parameter we can take $\tau_{0}=0$. If $\left(C_{k}^{\tau}, S_{k}^{\tau}\right) \in \mathbb{R}$ and $\left(C_{k}^{T}, S_{k}^{T}\right) \in$ $\mathbb{R}$ denote the Fourier real coefficients of $\tau$ and $\bar{T}$, respectively. Then from equation 47 it follows that for $k \neq 0$,

$$
C_{k}^{\tau}=\frac{1}{2}\left(C_{k}^{T}+S_{k}^{T} \cot \left(\frac{k \rho}{2}\right)\right), \quad S_{k}^{\tau}=\frac{1}{2}\left(S_{k}^{T}-C_{k}^{T} \cot \left(\frac{k \rho}{2}\right)\right) .
$$

As a final conclusion we can say that the two methods are closely related and one can easily go from one to the other through the return time. For the stroboscopic map method there are no geometric constraints in the selection of the invariant curve of the torus, which, on the other hand, introduces one more phase shift indetermination to deal with [10. In the Poincaré map method, the initial curve is specified by the intersection of the torus with a prescribed surface of section, this section needs to be carefully chosen since the vector field of the torus must be transverse to it. From the computational point of view, we can say that the Poincaré map method requires at each step of Newton's method the solution of $4\left(2 N_{f}+1\right) \times\left(4\left(2 N_{f}+1\right)-1\right)$ linear systems while in the stroboscopic map method the dimension is $6\left(2 N_{f}+1\right) \times\left(6\left(2 N_{f}+1\right)-1\right)$.

We prefer the stroboscopic map method over the Poincaré map method for our explorations because the reliance on geometry makes it difficult to choose a suitable Poincaré section guaranteeing the transversality of the tori with the section, especially during the continuation. 


\section{Acknowledgements}

Y.C. thanks the support of Doctorate Foundation of Northwestern Poly-

895

the discussion on parameterisation method, and to C. Peng for the generous share of his thesis and ideas. G.G. thanks the Catalan government for the grant 2014 SGR 1145, and MINECO-FEDER for the grants MTM2013-41168-P and MTM2016-80117-P. J.J.M. thanks MINECO-FEDER for the grant MTM2015-

[6] R. Barrio, F. Blesa, and S. Serrano: Bifurcations and chaos in Hamiltonian systems. International Journal of Bifurcation and Chaos, 20(5):1293-1319, 2010.

[1] E. Anderson, Z. Bai, et al.: LAPACK Users' Guide. Third edition. Society for Industrial and Applied Mathematics, 1999.

[2] J. Atchison, and M. Peck: Lorentz-augmented Jovian orbit insertion. Journal of Guidance, Control, and Dynamics, 32(2):418-425, 2009.

[3] N. Baresi, Z. P. Olikara, and D. J. Scheeres: Survey of numerical methods for computing quasi-periodic tori in Astrodynamics. In 26th AAS/AIAA Space Flight Mechanics Meeting, Napa, CA. 2016.

[4] R. Barrio, F. Blesa, and S. Serrano: Periodic, escape and chaotic orbits in the Copenhagen and the $(\mathrm{n}+1)$-body ring problems. Communications in Nonlinear Science and Numerical Simulation, 14(5):2229-2238, 2009.

[5] R. Barrio, and F. Blesa: Systematic search of symmetric periodic orbits in 2DOF Hamiltonian systems Chaos, Solitons \& Fractals, 41(2):560-582, 2009 
[7] B. Baudouy: "Heat transfer and cooling techniques at low temperature." arXiv preprint arXiv:1501.07153, 2015.

[8] L. S. Breger, P. Gurfil, K. T. Alfriend, S. R. Vadali, and J. P. How: Spacecraft formation flying. Elsevier Ltd., 2010.

[9] R. Broucke: Stability of periodic orbits in the elliptic, restricted three-body problem AIAA Journal, 7(6): 1003-1009, 1969

[10] G. Gómez, and J. M. Mondelo: The dynamics around the collinear equilibrium points of the RTBP. Physica D: Nonlinear Phenomena, 157(4):283$321,2001$.

[11] E. J. Doedel, R. C. Paffenroth, H. B. Keller, D. J. Dichmann, J. GalánVioque, and A. Vanderbauwhede: Continuation of periodic solutions in conservative systems with applications to the 3 -body problem. Int. J. Bifurcation Chaos Appl. Sci. Eng., 13:1-29, 2003.

[12] E. J. Doedel, B. W. Kooi, Y. A. Kuznetsov, and G. A. R. Van Voorn: Continuation of connecting orbits in 3D-ODEs (I and II): point-to-cycle connections. Int. J. Bifurcation Chaos Appl. Sci. Eng., 18:18891903, 2008, and 19:159-169, 2009.

[13] E. J. Doedel, B. E. Oldeman, et al.: AUTO-07P: Continuation and Bifurcation Software for Ordinary Differential Equations. Concordia University, Montreal, 2010. http://cmvl.cs.concordia.ca/auto/.

[14] G. Gómez, J. Llibre, R. Martínez, and C. Simó.: Dynamics and mission design near libration points. Vol. I Fundamentals: The case of collinear libration points. World Scientific, Singapore, 2001.

[15] G. Gómez, and M. Marcote: High-order analytical solutions of Hill's equations. Celestial Mechanics and Dynamical Astronomy, 94(2):197-211, 2006.

[16] À. Haro, M. Canadell, J. L. Figueras, A. Luque, and J. M.Mondelo: The parameterization method for invariant manifolds. Springer, 2016. 
[17] M. E. Hough: Lorentz force perturbations of a charged ballistic missile. Proceedings of the AIAA Guidance and Control Conference, AIAA-19821549, San Diego, CA, 1982.

[18] R. S. Irving: Integers, polynomials, and rings. Springer, 2004.

[19] À. Jorba, and J. Villanueva: On the persistence of lowerdimensional invariant tori under quasiperiodic perturbations. Journal of Nonlinear Science, 7:427473, 1997.

[20] L. B. King, G. G. Parker, S. Deshmukh, and J. H. Chong: Study of interspacecraft Coulomb forces and implications for formation flying. Journal of Propulsion and Power, 19(3):497-505, 2003.

[21] E. Kolemen, N. J. Kasdin, and P. Gurfil: Multiple Poincaré sections method for finding the quasiperiodic orbits of the restricted three body problem. Celestial Mechanics and Dynamical Astronomy, 112(1):47-74, 2012.

[22] E. Kong, D. Kwon, S. Schweighart, L. Elias, R. Sedwick, and D. Miller: Electromagnetic formation flight for multi-satellite arrays. Journal of Spacecraft and Rockets, 41(4):659-666, 2004.

[23] D. W. Kwon: Propellantless formation flight applications using electromagnetic satellite formations. Acta Astronautica, 67(9-10): 1189-1201, 2010.

[24] D. W. Kwon, and R. J. Sedwick: Cryogenic heat pipe for cooling hightemperature superconducting coils. Journal of Thermophysics and Heat Transfer, 23(4): 732-740, 2012.

[25] K. Meyer, G. Hall, and D. Offin: Introduction to Hamiltonian dynamical systems and the N-body problem. Springer Science \& Business Media, 2008.

[26] K. E. Papadakis, C. G. Zagouras. Bifurcation points and intersections of families of periodic orbits in the three-dimensional restricted three-body problem. Astrophysics and Space Science, 199(2): 241-256, 1993. 
[27] M. A. Peck: Prospects and challenges for Lorentz-augmented orbits. Proceedings of the AIAA Guidance, Navigation, and Control Conference, AIAA-2005-5995, San Francisco, CA, 2005.

[33] Y. Ren, J. J. Masdemont, M. Marcote, and G. Gómez: Computation of analytical solutions of the relative motion about a Keplerian elliptic orbit. Acta Astronautica, 81(1):186-199, 2012.

[34] C. M. Saaj, V. Lappas, D. Richie, M. Peck, B. Streetman, and H. Schaub: 995 Electrostatic forces for satellite swarm navigation and reconfiguration Final report. Final Report for Ariadna Study Id. AO491905, 2006.

[35] L. Schaffer, and J. A. Burns: Charged dust in planetary magnetospheres: Hamiltonian dynamics and numerical simulations for highly charged grains. Journal of Geophysical Research, 9(A9):17211-17223, 1994. 
[36] C. Simó: On the analytical and numerical approximation of invariant manifolds. In D. Benest and C. Froeshlé, editors, Modern methods in Celestial Mechanics: 285330. Editions Frontires, 1990.

[37] C. Skokos: On the stability of periodic orbits of high dimensional autonomous Hamiltonian systems Physica D: Nonlinear Phenomena, 159(3):155-179, 2001.

[38] L. A. Sobiesiak, and C. J. Damaren: Controllability of Lorentz-augmented spacecraft formations. Journal of Guidance Control and Dynamics, 38(11):2188-2195, 2015.

[39] J. Stoer, R. Bulirsch: Introduction to numerical analysis (Vol. 12). Springer Science \& Business Media, 2013.

[40] B. Streetman, and M. A. Peck: New synchronous orbits using the geomagnetic Lorentz force. Journal of Guidance, Control and Dynamics, 30(6):1677-1690, 2007.

[41] B. Streetman, and M. A. Peck: A General Bang-Bang Control Method for Lorentz Augmented Orbits. AAS Spaceflight Mechanics Meeting, AAS Paper 08-111, Galveston, Texas, 2008

[42] B. Streetman, and M. A. Peck: Gravity-assist maneuvers augmented by the Lorentz force. Journal of Guidance, Control, and Dynamics, 32(5):16391647, 2009.

[43] S. Tsujii, M. Bando, and H. Yamakawa: Spacecraft formation flying dynamics and control using the geomagnetic Lorentz force. Journal of Guidance, Control, and Dynamics, 36(1): 136-148, 2012.

[44] A. Umair, D. W. Miller, and J. L. Ramirez: Control of electromagnetic satellite formations in near-Earth orbit. Journal of Guidance, Control and Dynamics, 33(6):1883-1891, 2010. 
[45] D. Vokrouhlicky: The Geomagnetic effects on the motion of electrically charged artificial satellite, Celestial Mechanics \& Dynamical Astronomy, 46(1):85-104, 1989. 